\title{
NECESSARY STOCHASTIC MAXIMUM PRINCIPLE FOR DISSIPATIVE SYSTEMS ON INFINITE TIME HORIZON
}

\author{
CARLO ORRIERI AND PETR VEVERKA
}

\begin{abstract}
We develop a necessary stochastic maximum principle for a finite-dimensional stochastic control problem in infinite horizon under a polynomial growth and joint monotonicity assumption on the coefficients. The second assumption generalizes the usual one in the sense that it is formulated as a joint condition for the drift and the diffusion term. The main difficulties concern the construction of the first and second order adjoint processes by solving backward equations on an unbounded time interval. The first adjoint process is characterized as a solution to a backward SDE, which is well-posed thanks to a duality argument. The second one can be defined via another duality relation written in terms of the Hamiltonian of the system and linearized state equation. Some known models verifying the joint monotonicity assumption are discussed as well.
\end{abstract}

\section{INTRODUCTION}

The study of the stochastic maximum principle (SMP in short) is a current field of research motivated by the interest in finding necessary (and sufficient) conditions for optimality for stochastic control problems. The general idea of the SMP consists in associating to every controlled trajectory an adjoint equation which is backward in time. Its solution, called a dual process (which is, in fact, a pair of processes), is shown to exist under some appropriate conditions and plays a role of "generalised Lagrange multipliers". The SMP is a variational inequality formulated by means of the state trajectory and the dual process. It is satisfied by any optimal control and, usually, adding some convexity assumptions, it fully characterizes the optimality. Moreover, if the control enters the diffusion, the irregularity in time of the noise forces to introduce a second adjoint process which is strictly related to the Lyapunov equation for the first variation of the state.

The first general formulation of the SMP is due to Peng [17] for finite dimensional systems. After this seminal work, there has been a large number of works on this subject, both in finite and infinite dimensions for different formulation of the control problem. SMP in infinite dimension has been studied e.g. in Tang and Li [20], Fuhrman, Hu and Tessitore [7], Du and Meng [5], Lü and Zhang [9] whereas some of the results in finite dimension comprise: Jump control: Tang and Li 21], Øksendal and Sulem [1]]; Singular control: Bahlali and Mezerdi [1], Dufour and Miller 6], Øksendal and Sulem [12]; Impulse control: Wu and Zhang [23]; Delayed controlled systems: Øksendal, Sulem and Zhang [13]; Near-optimal control: Zhou [25] and many others.

This paper is a natural continuation of [10] on one side and [15] on the other. Our aim is to control the behaviour of a dissipative system in an unbounded time interval and to provide necessary conditions for optimality. If $W_{t}$ is a $d$-dimensional Brownian motion, the equation for the state can be written in the form

$$
X_{t}=x+\int_{0}^{t} b\left(X_{s}, u_{s}\right) d s+\int_{0}^{t} \sigma\left(X_{s}, u_{s}\right) d W_{s}
$$

$\mathbb{P}$-a.s. for all $t \in[0,+\infty)$ and all $x \in \mathbb{R}^{n}$. The objective is to minimize a discounted functional

$$
J(u(\cdot))=\mathbb{E} \int_{0}^{+\infty} e^{-r t} f\left(X_{t}, u_{t}\right) d t
$$

1991 Mathematics Subject Classification. Primary: 93E20, 60H10; Secondary: 49K45.

Key words and phrases. Stochastic maximum principle, dissipative systems, backward stochastic differential equation, stochastic discounted control problem, infinite time horizon, necessary conditions for optimality.

The first author has been supported by the Gruppo Nazionale per l'Analisi Matematica, la Probabilità e le loro Applicazioni (GNAMPA) of the Istituto Nazionale di Alta Matematica (INdAM)

The second author has been supported by the grant MIUR PRIN 2011 "Evolution differential problems: deterministic and stochastic approaches and their interactions". 
over all admissible controls $u(\cdot)$ with values in a general separable metric space $(U, d)$. Let us remark that this general assumption on the space of control actions allows us to consider a broad class of controls, such as bang-bang controls, which are excluded from the classic convexity framework. On the other hand, it necessarily forces us to formulate the SMP via a spike perturbation argument.

The analysis of the problem is based on the well-posedness of the state equation under a joint (or global) monotonicity assumption. For any $x, y \in \mathbb{R}^{n}$ and fixed $p>0$, there exists $c_{p} \in \mathbb{R}$ such that

$$
\langle b(x, u)-b(y, u), x-y\rangle+p\|\sigma(x, u)-\sigma(y, u)\|_{2}^{2} \leq c_{p}|x-y|^{2}, \quad \forall u \in U,
$$

where $U$ is the space of control actions. For a detailed exposition of SDEs with this property see [3]. This condition is a generalization of the usual dissipativity condition which involves only the drift of the equation and allows us to consider a larger class of concrete examples. Informally, there is a balance between the dissipativity of the drift and the noise term. If the drift term is dissipative enough, the diffusion term can grow in a polynomial way, instead of being globally Lipschitz. As a reference for this general assumption, see [3] and [19]. A list of several important examples satisfying joint monotonicity condition is given further in this paper. However, let us notice that many interesting equations do not satisfy a global monotonicity assumption (see [4] for a selection of examples) and the formulation of a version of the SMP for these systems could be a subject of a future work.

In the first step of our analysis we have to deal with a partially-coupled system of the state equation and the first adjoint equation. The delicate question here consists in giving a precise meaning to the solution of the following backward SDE

$$
d p_{t}=-\left[D_{x} H\left(\bar{X}_{t}, \bar{u}_{t}, p_{t}, q_{t}\right)-r p_{t}\right] d t+q_{t} d W_{t}
$$

where $H(x, u, p, q)=\langle p, b(x, u)\rangle+\operatorname{Tr}\left[q^{T} \sigma(x, u)\right]-f(x, u)$ is the Hamiltonian of the system and $(\bar{X}, \bar{u})$ is an optimal pair.

In general, the behaviour at infinity of BSDEs is not easy to understand and different approaches and approximations are proposed in several papers. In our setting, we are able to tackle the problem showing that the adjoint equation preserves, in some sense, the dissipativity of the state. Using a duality argument and the same technique as in [18] we can show that there exists a solution in some exponentially weighted space. It turns out that for the analysis of the state and adjoint equations the condition on the discount factor (forming both the functional and the weight $e^{-r t}$ in the definition of exponential weighted space) is given by some formula in terms of the joint monotonicity constant $c_{p} \in \mathbb{R}$. Nevertheless, due to the framework of SMP (use of the spike variation techniques) and, more importantly, due to the form of the polynomial growth assumption one has to assume implicitly $r$ positive so that the polynomial bound is integrable with the weight.

As already mentioned, the presence of the control in the diffusion term makes a second adjoint process to appear. In this case, there exists a formal matrix-valued BSDE which represents the process, but due to the lack of dissipativity of the equation, it seems to be impossible to obtain an a priori estimate of the solution. To solve the problem we follow the idea of Fuhrman et al. in [7] and we define the first component of the second adjoint process $P_{t}$ as a bilinear form defined via the relation

$$
\left\langle P_{t} \eta, \gamma\right\rangle:=\mathbb{E}^{\mathcal{F}_{t}} \int_{t}^{\infty} e^{-r(s-t)}\left\langle D_{x}^{2} H\left(\bar{X}_{s}, \bar{u}_{s}, p_{s}, q_{s}\right) y_{s}^{t, \eta}, y_{s}^{t, \gamma}\right\rangle d s
$$

where $\left(y_{s}^{t, \eta}\right)_{s \geq t}$ is the solution of the linearized state equation starting from $\eta$ at time $t$. Note that the second component $Q_{t}$ does not appear in the definition of the SMP. Proceeding this way it is not necessary to define and solve the second adjoint equation (i.e. finding the couple $(P, Q)$ ) but it is sufficient to identify only the process $P$ via the equality (3). Notice that our definition of $P$ is similar to the notion of transposition solution presented in [9]. Nevertheless, if the diffusion term $\sigma$ is Lipschitz, $P$ can be indeed identified as a unique solution to a matrix-valued BSDE which, in fact, inherits the monotonicity property from the state equation. The formulation of the SMP, in this case, follows by similar arguments as in [15] but with an extension to the infinite horizon setting.

Having in mind the form of the Hamiltonian of the system, the final step (and the main result, Theorem (5) is to derive a necessary condition for optimality. Let us suppose that $(\bar{X}, \bar{u})$ is an 
optimal pair, then for every $v \in U$, the following variational inequality has to hold $\mathbb{P} \otimes d t$-a.e.

$H\left(\bar{X}_{t}, v, p_{t}, q_{t}\right)-H\left(\bar{X}_{t}, \bar{u}_{t}, p_{t}, q_{t}\right)+\frac{1}{2} \sum_{j=1}^{d}\left\langle P_{t}\left(\sigma^{j}\left(\bar{X}_{t}, v\right)-\sigma^{j}\left(\bar{X}_{t}, \bar{u}_{t}\right)\right), \sigma^{j}\left(\bar{X}_{t}, v\right)-\sigma^{j}\left(\bar{X}_{t}, \bar{u}_{t}\right)\right\rangle \leq 0$.

This variational inequality can be rewritten in terms of so called $\mathcal{H}$-function defined by

$$
\begin{aligned}
\mathcal{H}(x, u):= & H\left(x, u, p_{t}, q_{t}\right)-\frac{1}{2} \operatorname{Tr}\left(\sigma\left(\bar{X}_{t}, \bar{u}_{t}\right)^{T} P_{t} \sigma\left(\bar{X}_{t}, \bar{u}_{t}\right)\right) \\
& +\frac{1}{2} \operatorname{Tr}\left[\left(\sigma(x, u)-\sigma\left(\bar{X}_{t}, \bar{u}_{t}\right)\right)^{T} P_{t}\left(\sigma(x, u)-\sigma\left(\bar{X}_{t}, \bar{u}_{t}\right)\right)\right],
\end{aligned}
$$

in the following manner

$$
\mathcal{H}\left(\bar{X}_{t}, \bar{u}_{t}\right)=\max _{v \in U} \mathcal{H}\left(\bar{X}_{t}, v\right), \quad \mathbb{P} \otimes d t-\text { a.e. }
$$

The paper is organized as follows. The second and third sections contain the basic assumptions, the formulation of the discounted problem and a list of motivating examples. In section 4 , we study well posedness of the state equation. The fifth section concerns with the application of the spike variation technique to our problem. In the two next sections, 6 and 7 , the two adjoint processes are studied. The first adjoint BSDE is solved by approximation and a duality argument whereas, the construction of the process $P$ is described without any potential relation to some BSDE. A precise statement of the main theorem (Theorem 5) is presented in section 8 and its proof is given. Last, in the Appendix we provide some technical proofs and we quote the actual restriction for the discount factor including the final one used in formulation of the main result.

\section{Assumptions And PRELiminaries}

Let $W=\left\{W_{t}^{1}, \ldots, W_{t}^{d}\right\}_{t \geq 0}$ be a standard $d$-dimensional Brownian motion defined on some complete filtered probability space $\left(\Omega, \mathcal{F},\left(\mathcal{F}_{t}\right)_{t \geq 0}, \mathbb{P}\right)$. The filtration $\left(\mathcal{F}_{t}\right)_{t \geq 0}$ is assumed to be the canonical filtration of $W$ completed by $\mathbb{P}$-null sets. The space of control actions is a general metric space $U$ endowed with its Borel $\sigma$-algebra $\mathcal{B}(U)$. The class of admissible controls is defined as follows

$$
\mathcal{U}:=\left\{u(\cdot): \mathbb{R}_{+} \times \Omega \rightarrow U: u(\cdot) \text { is }\left(\mathcal{F}_{t}\right)_{t \geq 0}-\text { progressive }\right\} .
$$

For $r \in \mathbb{R}, p>1$ and a Banach space $E$, we define

$$
\begin{aligned}
L_{\mathcal{F}}^{p,-r}\left(\mathbb{R}_{+} ; E\right):=\left\{v(\cdot): \mathbb{R}_{+}\right. & \times \Omega \rightarrow E: v(\cdot) \text { is }\left(\mathcal{F}_{t}\right)_{t \geq 0}-\text { progressive } \\
& \text { and } \left.\mathbb{E} \int_{0}^{\infty} e^{-r t}\left\|v_{t}\right\|_{E}^{p} d t<\infty\right\} .
\end{aligned}
$$

We want to study an infinite horizon stochastic control problem in $\mathbb{R}^{n}$ of the form

$$
\left\{\begin{array}{l}
d X_{t}=b\left(X_{t}, u_{t}\right) d t+\sigma\left(X_{t}, u_{t}\right) d W_{t}, \quad \forall t \geq 0 \\
X_{0}=x
\end{array}\right.
$$

where $x \in \mathbb{R}$ and $u(\cdot)$ is an admissible control. The discounted functional to be minimized is given by

$$
J(u(\cdot))=\mathbb{E} \int_{0}^{+\infty} e^{-r t} f\left(X_{t}, u_{t}\right) d t .
$$

By $|\cdot|$ we denote the Euclidean norm on $\mathbb{R}^{n},\|\cdot\|$ stands for a Frobenius norm on $\mathbb{R}^{n \times d}$ and, finally, $\|\cdot\|_{2}$ denotes the Hilbert-Schmidt norm on $\mathbb{R}^{n \times n}$. By $\mathcal{S}^{n}$ we denote the set of symmetric matrixes $\mathbb{R}^{n \times n} \cdot \chi_{A}$ denotes the characteristic function of a set $A$.

\section{Hypotheses:}

(H1) $(U, d)$ is a separable metric space. 
(H2) (Polynomial growth) The vector field $b: \mathbb{R}^{n} \times U \rightarrow \mathbb{R}^{n}$ is $\mathcal{B}\left(\mathbb{R}^{n}\right) \otimes \mathcal{B}(U)$-measurable and the map $x \mapsto b(x, u)$ is of class $\mathcal{C}^{2}$. Moreover, there exists $m \geq 0$ such that

$$
\sup _{u \in U} \sup _{x \in \mathbb{R}^{n}} \frac{\left|D_{x}^{\beta} b(x, u)\right|}{1+|x|^{2 m+1}}<+\infty, \quad|\beta|=0,1,2 .
$$

(H3) (Polynomial growth) The mapping $\sigma: \mathbb{R}^{n} \times U \rightarrow \mathbb{R}^{n \times d}$ is measurable with respect to $\mathcal{B}\left(\mathbb{R}^{n}\right) \otimes \mathcal{B}(U)$. Moreover the map $x \mapsto \sigma(t, x, u)$ is $\mathcal{C}^{2}\left(\mathbb{R}^{n} ; \mathbb{R}^{n \times d}\right)$ and there exists $m$ (same as in (H2)) such that

$$
\sup _{u \in U} \sup _{x \in \mathbb{R}^{n}} \frac{\left\|D_{x}^{\beta} \sigma(x, u)\right\|_{2}}{1+|x|^{m}}<+\infty, \quad|\beta|=0,1,2 .
$$

(H4) (Joint monotonicity) Let $p>0$. Then there exists $c_{p} \in \mathbb{R}$ such that,

$$
\left\langle D_{x} b(x, u) y, y\right\rangle+p\left\|D_{x} \sigma(x, u) y\right\|_{2}^{2} \leq c_{p}|y|^{2}, \quad x, y \in \mathbb{R}^{n}, u \in U
$$

(H5) (Cost) The function $f: \mathbb{R}^{n} \times U \rightarrow \mathbb{R}$ is $\mathcal{B}\left(\mathbb{R}^{n}\right) \otimes \mathcal{B}(U)$-measurable and the map $x \mapsto f(x, u)$ is of class $\mathcal{C}^{2}$. Moreover, there exists $l \geq 0$ such that

$$
\sup _{u \in U} \sup _{x \in \mathbb{R}^{n}} \frac{\left|D_{x}^{\beta} f(x, u)\right|}{1+|x|^{l}}<+\infty, \quad|\beta|=0,1,2 .
$$

\section{Remark 1.}

(a) In [3], the form of (H2) and (H3) is given in a stronger way. For our purposes the above formulation is sufficient.

(b) It is possible to show that condition (91) implies that for any $x, y \in \mathbb{R}^{n}$

$$
\langle b(x, u)-b(y, u), x-y\rangle+p\|\sigma(x, u)-\sigma(y, u)\|_{2}^{2} \leq c_{p}|x-y|^{2}, \quad u \in U .
$$

and the two conditions are equivalent for coefficients twice differentiable (in $x$ ) which, in fact, is our case.

(c) The joint monotonicity condition (111) also implies the so called coercivity condition (see i.e. [19], page 44). Indeed, let us fix $y=0$, then (11) reduces to

$$
\langle b(x, u)-b(0, u), x\rangle+p\|\sigma(x, u)-\sigma(0, u)\|_{2}^{2} \leq c_{p}|x|^{2},
$$

and

$$
\begin{aligned}
\|\sigma(x, u)-\sigma(0, u)\|_{2}^{2} & \geq\left|\|\sigma(x, u)\|_{2}-\|\sigma(0, u)\|_{2}\right|^{2} \\
& =\|\sigma(x, u)\|_{2}^{2}+\|\sigma(0, u)\|_{2}^{2}-2\|\sigma(x, u)\|_{2}\|\sigma(0, u)\|_{2} \\
& \geq\|\sigma(x, u)\|_{2}^{2}+\|\sigma(0, u)\|_{2}^{2}-\varepsilon\|\sigma(x, u)\|_{2}^{2}-\frac{\|\sigma(0, u)\|_{2}^{2}}{\varepsilon} \\
& =(1-\varepsilon)\|\sigma(x, u)\|_{2}^{2}+\|\sigma(0, u)\|_{2}^{2}-\frac{\|\sigma(0, u)\|_{2}^{2}}{\varepsilon}, \forall \varepsilon \in(0,1) .
\end{aligned}
$$

Then after easy computation we obtain that

$$
\langle b(x, u), x\rangle+p(1-\varepsilon)\|\sigma(x, u)\|_{2}^{2} \leq \tilde{K}_{p}\left(1+|x|^{2}\right), \quad \varepsilon \in(0,1) .
$$

Let us note that $|b(0, u)|+|\sigma(0, u)| \leq C$ due to the polynomial growth (H2)-(H3), hence $\tilde{K}_{p}$ can be chosen as $\tilde{K}_{p}=\max \left\{c_{p}+1 / 2, C^{2} / 2,1\right\}$.

(d) The above Hypotheses (H2)-(H5) can be generalized to the situation of time dependent stochastic coefficients under natural assumptions without any influence on the main result. 


\section{Motivations And EXAmples}

Apart from the classical Lipschitz setting, there are two usual sets of assumptions which assure global existence and uniqueness of the solution to an SDE. The first one consists in local Lipschitz property of the coefficients along with the so called coercivity condition

$$
\langle b(x), x\rangle+\frac{1}{2}\|\sigma(x)\|_{2}^{2} \leq K\left(1+|x|^{2}\right),
$$

for all $x \in \mathbb{R}^{n}$ and some $K \in \mathbb{R}$. The second set comprises some dissipativity assumptions on the drift, still with Lipschitz diffusion term. The dissipativity is expressed by

$$
\langle b(x)-b(y), x-y\rangle \leq K|x-y|^{2},
$$

for all $x, y \in \mathbb{R}^{n}$ and some $K \in \mathbb{R}$.

Another step further in this direction is the so called global monotonicity assumption, which is formulated as a joint condition for drift and diffusion

$$
\langle b(x)-b(y), x-y\rangle+\frac{1}{2}\|\sigma(x)-\sigma(y)\|_{2}^{2} \leq c|x-y|^{2},
$$

for all $x, y \in \mathbb{R}^{n}$. It is important to mention that the joint monotonicity property immediately implies the dissipativity of the drift but not necessarily global Lipschitzianity of the diffusion part. Moreover, the joint monotonicity (15) also implies the coercivity property (14).

It turns out that for the purposes of the SMP it is natural to strengthen the global monotonicity assumption in the following form: for all fixed $p>0$ there exists $c_{p} \in \mathbb{R}$ such that

$$
\langle b(x)-b(y), x-y\rangle+p\|\sigma(x)-\sigma(y)\|_{2}^{2} \leq c_{p}|x-y|^{2},
$$

for all $x, y \in \mathbb{R}^{n}$. This is motivated by the attempt to solve not only the state equation, but also the first and second variation equations and to derive some appropriate estimates of higher moments of the solutions. Another natural assumption in this framework is the polynomial growth of the coefficients along with their derivatives. This is fundamental in order to choose the correct discount factor in the definition of the weighted spaces $L_{\mathcal{F}}^{2,-r}\left(\mathbb{R}_{+} ; \mathbb{R}^{n}\right)$ that we are going to use. Let us also mention that these polynomial bounds allow us to prove the local Lipschitzianity of the coefficients of the state equation.

To conclude, notice that the freedom in choosing $p$ in the definition of (16) implies the existence of a link between the growth of the diffusion term and the drift (compare (H2) and (H3), see also [3]). For example, to gain a quadratic growth in the diffusion we have to require the system to be more dissipative. Concretely, one such an example is

$$
d X_{t}=\left[X_{t}-X_{t}^{5}\right] d t+X_{t}^{2} d W_{t} .
$$

A more general framework is presented in [19] and [8] where the authors do not ask for polynomial growth of the coefficients and present a weak local version of the global monotonicity assumption along with a weak coercivity assumption. By weak we mean the presence of stochastic coefficients instead of constant ones in the definitions of the conditions, see [19] for a detailed exposure.

After this preliminary discussion we also present some concrete models.

(1) Polynomial model: As we have discussed above, a one dimensional model given by the SDE

$$
d X_{t}=\left[-X_{t}^{2 m+1}+\sum_{i=1}^{2 m} a_{i} X_{t}^{i}\right] d t+\left[\sum_{i=1}^{m} b_{i} X_{t}^{i}\right] d W_{t} ; \quad X_{0}=x \in \mathbb{R},
$$

for some $a_{i}, b_{i} \in \mathbb{R}$, is the simplest example we have in mind. Let us notice that we can easily generalize the model in a way so that these polynomials are upper bounds for some more general (but locally Lipschitz) functions satisfying the joint monotonicity condition.

(2) Population growth models: A model in $\mathbb{R}$ given by the SDE

$$
d X_{t}=\alpha X_{t} h\left(X_{t}\right) d t+\sigma X_{t} d W_{t} ; \quad X_{0}=x>0,
$$

where $h(x)=1-\beta \ln (x)$, for so called Gompertz growth models (tumor growth models etc.) or $h(x)=1-\beta x$, for so called logistic growth models (population dynamics models etc.). A detailed discussion of the controlled logistic model on infinite time horizon can 
be found [10]. In both cases, $\alpha>0$ is the speed of growth and $\beta>0$ represents some saturation level of the system. It can be shown by Lyapunov techniques that the solution is positive and an explicitly analytic formula can be found by linearizing the two equations. It is important to mention that our version of SMP covers the case of controlled logistic models (in full generality) whereas the controlled Gompertz model can be treated only with uncontrolled diffusion. This fact is due to the lack of polynomial growth condition needed in (H2) and the second variation equation might not be well posed. The same argument holds for another generalizations of the two population models with different choices of diffusion term $(\sigma x(1-\ln (x)), \sigma \sqrt{x(1-\ln (x))}, \sigma \sqrt{x(1-x)}$ etc.).

(3) Gradient flow model with stochastic perturbation: Let $K$ be a convex open subset of $\mathbb{R}^{d}$ and $\varphi: K \subseteq \mathbb{R}^{d} \rightarrow \mathbb{R}$ a $\lambda$-convex function, i.e.

$$
\varphi\left((1-\alpha) x_{0}+\alpha x_{1}\right) \leq(1-\alpha) \varphi\left(x_{0}\right)+\alpha \varphi\left(x_{1}\right)-\frac{\lambda}{2} \alpha(\alpha-1)\left|x_{1}-x_{0}\right|^{2},
$$

for every $x_{0}, x_{1} \in \mathbb{R}^{d}$ and $\alpha \in[0,1]$. Then we can study a SDE of the form

$$
d X_{t}=-\nabla \varphi\left(X_{t}\right) d t+\sigma\left(X_{t}\right) d W_{t},
$$

provided that $\varphi$ is of class $C^{1}$. In fact, $\lambda$-convexity (with $C^{1}$-regularity) is equivalent to

$$
\langle\nabla \varphi(y), y-x\rangle-\frac{\lambda}{2}|y-x|^{2} \geq \varphi(y)-\varphi(x) \geq\langle\nabla \varphi(x), y-x\rangle+\frac{\lambda}{2}|y-x|^{2}
$$

which in particular implies that $\nabla \varphi$ is $\lambda$-dissipative. If we ask $\sigma$ to be Lipschitz, then (11) is satisfied.

Some possible choices of $\varphi(\cdot)$ are:

- Take $\lambda=0$ and $\varphi(x)=|x|^{2 k}$ convex with the derivative $2 k|x|^{2 k-2} x$;

- (Double-well potential) Let $d=1$ and consider $\varphi(x)=\left(x^{2}-1\right)^{2}$, which is not convex ( \pm 1 are minima) but $\lambda$-convex.

- Let $d=2$ and consider the following dynamics

$$
\left\{\begin{array}{l}
d X_{t}=-X_{t} d t+X_{t} Y_{t}^{2}\left(1+X_{t}^{2}\right)^{-2} d t+\sigma d W_{t}^{(1)}, \\
d Y_{t}=-Y_{t}\left(1+X_{t}^{2}\right)^{-1} d t+\sigma d W_{t}^{(2)} .
\end{array}\right.
$$

Here the energy has the following form $\varphi=\varphi_{1}+\varphi_{2}$, where

$$
\varphi_{1}(x, y)=\frac{x^{2}}{2} \quad \text { and } \quad \varphi_{2}(x, y)=\frac{y^{2}}{2\left(1+x^{2}\right)} .
$$

The difference between this case and the previous one is that here, the energy has not isolated minima but rather forms a sub-manifold (i.e. the $x$-axis).

\section{State Equation}

In this section we provide the existence and uniqueness theorem for the state equation (5). The classical proof of such theorem under our Hypotheses (H1)-(H4) goes along the lines as in [3], Section 1.2. for a solution in the space $L_{\mathcal{F}}^{2}\left([0, T] ; \mathbb{R}^{n}\right.$ ) (thus not in exponentially weighted space $\left.L_{\mathcal{F}}^{2,-r}\left([0, T] ; \mathbb{R}^{n}\right)\right)$. Nevertheless, by these arguments one can not obtain a contraction from $L_{\mathcal{F}}^{2,-r}\left([0,+\infty) ; \mathbb{R}^{n}\right)$ to itself (not even locally in time). Hence an another approach has to be chosen. More specifically, we will scale the original equation in a way so that the classical result from [3] can be applied. We stress that, in this case, the bound for the discount factor depends on $c_{p}$ which can be also negative.

Theorem 1. Let Hypotheses (H1)-(H4) hold. Then for every admissible control $u(\cdot)$ there is a unique solution process $\left(X_{t}\right)_{t \geq 0}$ to $S D E$ (5) with $\sup _{t \in[0, T]} \mathbb{E}\left[e^{-r t}\left|X_{t}\right|^{2}\right]<+\infty$, for each $T>0$ and for $r>2 c_{1 / 2}$. Moreover, for all $q \geq 2$ and for $r>2 c_{q-1}$ it holds

$$
\mathbb{E} \int_{0}^{\infty} e^{-r t q}\left|X_{t}\right|^{2 q} d t \leq C_{1}|x|^{2 q}, \quad \text { for some constant } C_{1}=C_{1}(q)>0
$$


where $c_{q-1}$ is the joint monotonicity constant in (9). In addition, for $q \in\left[\frac{1}{2}, 2\right)$ and $r>4 c_{1}$, it holds

$$
\mathbb{E} \int_{0}^{\infty} e^{-r t}\left|X_{t}\right|^{2 q} d t \leq C_{2}|x|^{4}, \quad \text { for some constant } C_{2}=C_{2}(q)>0 .
$$

Proof. Without loss of generality we prove the result for uncontrolled case (which can be easily converted to the controlled one). The idea is to apply the result from [3] to a transformed equation which corresponds to our original equation. Assume for a moment that we already have a process $X$ satisfying the dynamics given by (5) and define $\tilde{X}_{t} \equiv e^{-\frac{r}{2} t} X_{t}$. Then $\tilde{X}$ solves

$$
\left\{\begin{array}{l}
d \tilde{X}_{t}=-\frac{r}{2} \tilde{X}_{t}+e^{-\frac{r}{2} t} b\left(e^{\frac{r}{2} t} \tilde{X}_{t}\right) d t+e^{-\frac{r}{2} t} \sigma\left(e^{\frac{r}{2} t} \tilde{X}_{t}\right) d W_{t}, \quad \forall t \geq 0, \\
\tilde{X}_{0}=x
\end{array}\right.
$$

Denoting $\tilde{b}(t, x)=-\frac{r}{2} x+e^{-\frac{r}{2} t} b\left(e^{\frac{r}{2} t} x\right)$ and $\tilde{\sigma}(t, x)=e^{-\frac{r}{2} t} \sigma\left(e^{\frac{r}{2} t} x\right)$ it is easy to check that $\tilde{b}, \tilde{\sigma}$ also satisfy assumptions (H1)-(H4). Differentiability and polynomial growth (H2)-(H3) are evident whereas joint monotonicity (H4) holds in the following sense

$$
\langle\tilde{b}(t, x)-\tilde{b}(t, y), x-y\rangle+p\|\tilde{\sigma}(t, x)-\tilde{\sigma}(t, y)\|_{2}^{2} \leq \tilde{c}_{p}|x-y|^{2}, \quad \tilde{c}_{p}=c_{p}-\frac{r}{2} .
$$

Hence, due to [3], Section 1.2 there exists a unique predictable process $\tilde{X}$ solving (20) which satisfies $\sup _{t \in[0, T]} \mathbb{E}\left|\tilde{X}_{t}\right|^{2}<+\infty$, for each $T>0$. But this means that there actually exists a process $X$ solving (5) with the following integrability property

$$
\sup _{t \in[0, T]} \mathbb{E}\left[e^{-r t}\left|X_{t}\right|^{2}\right]=\sup _{t \in[0, T]} \mathbb{E}\left|\tilde{X}_{t}\right|^{2}<+\infty, \quad \forall T>0 .
$$

The last step is proving the desired exponentially weighted integrability. Denote the exit time $\tau_{K}:=\inf \left\{t \geq 0:\left|\tilde{X}_{t}\right| \geq K\right\}$ for each $K>0$ with the usual convention $\inf \{\emptyset\}:=+\infty$. It is easy to show that $\tau_{K} \nearrow \tau=+\infty$, for $K \rightarrow+\infty$ a.s.

Proof of estimate (18): Let us fix $q \geq 2$ and apply the Itô formula to $\left|\tilde{X}_{t \wedge \tau_{K}}\right|^{2 q}$. We also denote $\tilde{a}_{s}=\tilde{\sigma}\left(s, \tilde{X}_{s}\right) \tilde{\sigma}\left(s, \tilde{X}_{s}\right)^{T}$. Then we obtain

$$
\begin{aligned}
\mathbb{E}\left|\tilde{X}_{t \wedge \tau_{K}}\right|^{2 q}= & |x|^{2 q}+2 q \mathbb{E} \int_{0}^{t} \chi_{\left\{\tau_{K} \geq s\right\}}\left|\tilde{X}_{s}\right|^{2(q-1)}\left(\left\langle\tilde{X}_{s}, \tilde{b}\left(s, \tilde{X}_{s}\right)\right\rangle+\frac{1}{2} \operatorname{Tr}\left\{\tilde{a}_{s}\right\}\right) d s \\
& +2 q(q-1) \mathbb{E} \int_{0}^{t} \chi_{\left\{\tau_{K} \geq s\right\}}\left|\tilde{X}_{s}\right|^{2(q-2)} \operatorname{Tr}\left\{\tilde{a}_{s}\left(\tilde{X}_{s} \otimes \tilde{X}_{s}\right)\right\} d s \\
\leq & |x|^{2 q}+2 q \tilde{K}_{q-1} \tilde{c}_{q-1} \mathbb{E} \int_{0}^{t} \chi_{\left\{\tau_{K} \geq s\right\}}\left(\left|\tilde{X}_{s}\right|^{2 q}+1\right) d s \\
= & |x|^{2 q}+2 q \tilde{K}_{q-1} \tilde{c}_{q-1}\left(t \wedge \tau_{K}\right)+2 q \tilde{K}_{q-1} \tilde{c}_{q-1} \int_{0}^{t} \mathbb{E}\left|\tilde{X}_{s \wedge \tau_{K}}\right|^{2 q} d s,
\end{aligned}
$$

where we have used the joint monotonicity in the form of (11) and coercivity-type estimate (13). By Gronwall lemma it easily follows that

$$
\mathbb{E}\left|\tilde{X}_{t \wedge \tau_{K}}\right|^{2 q} \leq\left(|x|^{2 q}+2 q \tilde{K}_{q-1} \tilde{c}_{q-1}\left(t \wedge \tau_{K}\right)\right) e^{2 q \tilde{K}_{q-1} \tilde{c}_{q-1} t} \leq|x|^{2 q} e^{2 q \tilde{K}_{q-1}\left(c_{q-1}-\frac{r}{2}\right) t} .
$$

The last estimate can be made for $r \geq 2 c_{q-1}$. Consequently, the final estimate reads

$$
\mathbb{E}\left|\tilde{X}_{t}\right|^{2 q} \leq|x|^{2 q} e^{2 q \tilde{K}_{q-1}\left(c_{q-1}-\frac{r}{2}\right) t},
$$

and it follows by Fatou. Expressed in terms of the process $X$ we have that for all $t \geq 0$ it holds

$$
\mathbb{E}\left[e^{-r t q}\left|X_{t}\right|^{2 q}\right] \leq|x|^{2 q} e^{2 q \tilde{K}_{q-1}\left(c_{q-1}-\frac{r}{2}\right) t} .
$$

Now it is sufficient to integrate both sides of (24) on $[0,+\infty)$. 
Proof of estimate (19): Fix $q \geq 2$ and observe that $e^{-r t}\left|X_{t}\right|^{2 q}=e^{r t(q-1)}\left|\tilde{X}_{t}\right|^{2 q}$. Therefore, applying Itô formula to $e^{r t(q-1)}\left|\tilde{X}_{t}\right|^{2 q}$ gives

$$
\begin{aligned}
\mathbb{E}\left[e^{r(q-1)\left(t \wedge \tau_{K}\right)}\left|\tilde{X}_{t \wedge \tau_{K}}\right|^{2 q}\right]=|x|^{2 q}+2 q \mathbb{E} \int_{0}^{t} \chi_{\left\{\tau_{K} \geq s\right\}} e^{r(q-1) s}\left|\tilde{X}_{s}\right|^{2(q-1)} \times \\
\quad \times\left(\left\langle\tilde{X}_{s}, \tilde{b}\left(s, \tilde{X}_{s}\right)\right\rangle+\frac{1}{2} \operatorname{Tr}\left\{\tilde{a}_{s}\right\}\right) d s \\
\quad+2 q(q-1) \mathbb{E} \int_{0}^{t} \chi_{\left\{\tau_{K} \geq s\right\}} e^{r(q-1) s}\left|\tilde{X}_{s}\right|^{2(q-2)} \operatorname{Tr}\left\{\tilde{a}_{s}\left(\tilde{X}_{s} \otimes \tilde{X}_{s}\right)\right\} d s \\
\quad+r(q-1) \mathbb{E} \int_{0}^{t} \chi_{\left\{\tau_{K} \geq s\right\}} e^{r(q-1) s}\left|\tilde{X}_{s}\right|^{2 q} d s \\
\leq|x|^{2 q}+2 q \tilde{K}_{q-1} \tilde{c}_{q-1} \mathbb{E} \int_{0}^{t} \chi_{\left\{\tau_{K} \geq s\right\}} e^{r(q-1) s}\left(\left|\tilde{X}_{s}\right|^{2 q}+1\right) d s \\
\quad+r(q-1) \mathbb{E} \int_{0}^{t} \chi_{\left\{\tau_{K} \geq s\right\}} e^{r(q-1) s}\left|\tilde{X}_{s}\right|^{2 q} d s \\
\leq|x|^{2 q}+2 q \tilde{K}_{q-1} \tilde{c}_{q-1}\left(t \wedge \tau_{K}\right)+\left(2 q \tilde{K}_{q-1} \tilde{c}_{q-1}+r(q-1)\right) \mathbb{E} \int_{0}^{t} e^{r(q-1)\left(s \wedge \tau_{K}\right)}\left|\tilde{X}_{s \wedge \tau_{K}}\right|^{2 q} d s
\end{aligned}
$$

Then, similarly as before, we obtain

$$
\mathbb{E} \int_{0}^{\infty} e^{-r t}\left|X_{t}\right|^{2 q} d t \leq C_{2}|x|^{2 q}, \quad \text { for } r>2 q \tilde{K}_{q-1} c_{q-1},
$$

To conclude the proof observe that once we have obtained the estimates (25) for $q \geq 2$, the case $q \in[1 / 2,2)$ easily follows by Hölder inequality. Note that we have proved even more than stated in the assertion of the theorem. Nevertheless, such generality is not needed for the purposes of proving the SMP.

\section{SPIKE VARIATION AND VARIATION EQUATIONS}

The derivation of the variational inequality needed for the formulation of SMP is based on expanding the difference of the functional $J\left(u^{\varepsilon}(\cdot)\right)-J(\bar{u}(\cdot))$ where $\bar{u}(\cdot)$ is an optimal control and $u^{\varepsilon}(\cdot)$ is its appropriate perturbation. Since the control variable is allowed to enter also the diffusion term, the expansion has to be carried out up to second order due to the time irregularity of the noise. Therefore, two forward variation equations appear in our setting: first order variaton process $y^{\varepsilon}$ being, in fact, a linearization of the state process, and the second order variation process $z^{\varepsilon}$ coming from the second order expansion. We also stress that due to the estimation techniques used in the forthcoming proofs, we often need the polynomial bound of coefficients to be integrable with the weight, which immediately implies that $r$ has to be a priori positive.

Let $\varepsilon>0, E_{\varepsilon} \subset \mathbb{R}_{+}$be a set of measure $\varepsilon$ of the form $E_{\varepsilon}:=\left[t_{0}, t_{0}+\varepsilon\right]$, with $t_{0} \geq 0$ arbitrary but fixed, and $\bar{u}(\cdot)$ an optimal control. Define the spike variation $u^{\varepsilon}(\cdot)$ of $\bar{u}(\cdot)$ by the formula

$$
u_{t}^{\varepsilon}= \begin{cases}\bar{u}_{t}, & \text { if } t \in \mathbb{R}_{+} \backslash E_{\varepsilon}, \\ v, & \text { if } t \in E_{\varepsilon},\end{cases}
$$

where $v \in U$ is an arbitrary and fixed point.

Let $(\bar{X}(\cdot), \bar{u}(\cdot))$ be a given optimal pair and $\left(X^{\varepsilon}(\cdot), u^{\varepsilon}(\cdot)\right)$ satisfy the following perturbed SDE

$$
\left\{\begin{array}{l}
d X_{t}^{\varepsilon}=b\left(X_{t}^{\varepsilon}, u_{t}^{\varepsilon}\right) d t+\sigma\left(X_{t}^{\varepsilon}, u_{t}^{\varepsilon}\right) d W_{t} \\
X_{0}^{\varepsilon}=x
\end{array}\right.
$$

Further, following the notation of Yong and Zhou [24], we denote by $\delta \varphi_{t}$ the quantity $\varphi\left(\bar{X}_{t}\right.$, $\left.u_{t}^{\varepsilon}\right)-\varphi\left(\bar{X}_{t}, \bar{u}_{t}\right)$, for a generic function $\varphi$.

Now, let us begin studying the first variation equation

$$
\left\{\begin{array}{l}
d y_{t}^{\varepsilon}=D_{x} b\left(\bar{X}_{t}, \bar{u}_{t}\right) y_{t}^{\varepsilon} d t+\sum_{j=1}^{d}\left[D_{x} \sigma^{j}\left(\bar{X}_{t}, \bar{u}_{t}\right) y_{t}^{\varepsilon}+\delta \sigma_{t}^{j}\right] d W_{t}^{j}, \\
y_{0}^{\varepsilon}=0
\end{array}\right.
$$

and the second variation equation 


$$
\left\{\begin{aligned}
d z_{t}^{\varepsilon}= & {\left[D_{x} b\left(\bar{X}_{t}, \bar{u}_{t}\right) z_{t}^{\varepsilon}+\delta b_{t} \chi_{E_{\varepsilon}}(t)+\frac{1}{2} D_{x}^{2} b\left(\bar{X}_{t}, \bar{u}_{t}\right)\left(y_{t}^{\varepsilon}\right)^{2}\right] d t } \\
& +\sum_{j=1}^{d}\left[D_{x} \sigma^{j}\left(\bar{X}_{t}, \bar{u}_{t}\right) z_{t}^{\varepsilon}+\delta\left(D_{x} \sigma_{t}^{j}\right) y_{t}^{\varepsilon} \chi_{E_{\varepsilon}}(t)+\frac{1}{2} D_{x}^{2} \sigma^{j}\left(\bar{X}_{t}, \bar{u}_{t}\right)\left(y_{t}^{\varepsilon}\right)^{2}\right] d W_{t}^{j}, \\
z_{0}^{\varepsilon}= & 0
\end{aligned}\right.
$$

where we have adopted the notation

$$
\begin{gathered}
D_{x}^{2} b\left(\bar{X}_{t}, \bar{u}_{t}\right)\left(y_{t}^{\varepsilon}\right)^{2}:=\left(\begin{array}{c}
\operatorname{Tr}\left[D_{x}^{2} b^{1}\left(\bar{X}_{t}, \bar{u}_{t}\right) y_{t}^{\varepsilon}\left(y_{t}^{\varepsilon}\right)^{T}\right] \\
\vdots \\
\operatorname{Tr}\left[D_{x}^{2} b^{n}\left(\bar{X}_{t}, \bar{u}_{t}\right) y_{t}^{\varepsilon}\left(y_{t}^{\varepsilon}\right)^{T}\right]
\end{array}\right), \\
D_{x}^{2} \sigma^{j}\left(\bar{X}_{t}, \bar{u}_{t}\right)\left(y_{t}^{\varepsilon}\right)^{2}:=\left(\begin{array}{c}
\operatorname{Tr}\left[D_{x}^{2} \sigma^{1 j}\left(\bar{X}_{t}, \bar{u}_{t}\right) y_{t}^{\varepsilon}\left(y_{t}^{\varepsilon}\right)^{T}\right] \\
\vdots \\
\operatorname{Tr}\left[D_{x}^{2} \sigma^{n j}\left(\bar{X}_{t}, \bar{u}_{t}\right) y_{t}^{\varepsilon}\left(y_{t}^{\varepsilon}\right)^{T}\right]
\end{array}\right) .
\end{gathered}
$$

Theorem 2. Let Hypotheses (H1)-(H4) hold. Then there exist $r_{1} \in \mathbb{R}, r_{2}>0$ such that equation (27) (equation (28), resp.) admits a unique solution $y^{\varepsilon} \in L_{\mathcal{F}}^{2,-r_{1}}\left(\mathbb{R}_{+} ; \mathbb{R}^{n}\right)\left(z^{\varepsilon} \in L_{\mathcal{F}}^{2,-r_{2}}\left(\mathbb{R}_{+} ; \mathbb{R}^{n}\right)\right.$, resp.) for all admissible controls $u(\cdot) \in \mathcal{U}$.

Proof. Note first that (27) and (28) are linear equations in $y^{\varepsilon}$ and $z^{\varepsilon}$, respectively. The perturbation of the diffusion in (27) belongs to $L_{\mathcal{F}}^{2,-r}\left(\mathbb{R}_{+} ; \mathbb{R}^{n}\right)$ for every $r$, and it is independent on $y^{\varepsilon}$. Therefore, the joint monotonicity condition (11) holds and the proof follows by similar arguments as the proof of Theorem 1, for $r_{1}>2 c_{1 / 2}$. Concerning the equation for $z^{\varepsilon}$, we have to choose $r_{2}$ such that $D_{x}^{2} b\left(\bar{X}_{t}, \bar{u}_{t}\right)\left(y_{t}^{\varepsilon}\right)^{2}, D_{x}^{2} \sigma^{j}\left(\bar{X}_{t}, \bar{u}_{t}\right)\left(y_{t}^{\varepsilon}\right)^{2} \in L_{\mathcal{F}}^{2,-r_{2}}\left(\mathbb{R}_{+} ; \mathbb{R}^{n}\right)$. Then existence and uniqueness of a solution follow.

Remark 2. Let us note that, thanks to the linearity of the equation and due to the structure of the forcing term $\delta \sigma^{j}$, the solution $y^{\varepsilon}$ to the equation (27) is identically zero for times $t \leq t_{0}$.

In the rest of this section, we will often benefit from a general estimate of the solution to a linearized SDE given by the following Lemma.

Lemma 1. Let $Y \in L_{\mathcal{F}}^{2,-r}\left(\mathbb{R}_{+} ; \mathbb{R}^{n}\right)$ be a solution to the following linear $S D E$

$$
\left\{\begin{aligned}
d Y_{t} & =\left(A_{t} Y_{t}+\alpha_{t}\right) d t+\sum_{j=1}^{d}\left(B_{t}^{j} Y_{t}+\beta_{t}^{j}\right) d W_{t}^{j} \\
Y_{0} & =y_{0}
\end{aligned}\right.
$$

where $y_{0} \in \mathbb{R}^{n} ; A, B^{j}: \mathbb{R}_{+} \times \Omega \rightarrow \mathbb{R}^{n \times n}, \alpha, \beta^{j}: \mathbb{R}_{+} \times \Omega \rightarrow \mathbb{R}^{n}, j=1, \ldots, d$, all are $\left(\mathcal{F}_{t}\right)-$ progressively measurable processes. Let $k \geq 1, p>0$ and $c_{p} \in \mathbb{R}$ such that

1. $\left\langle A_{t} Y_{t}, Y_{t}\right\rangle+p \sum_{j=1}^{d}\left|B^{j} Y_{t}\right|^{2} \leq c_{p}\left|Y_{t}\right|^{2}, \quad \mathbb{P} \otimes d t-a . e$.

2. $\int_{0}^{\infty}\left[e^{-\frac{r}{2} t}\left(\mathbb{E}\left|\alpha_{t}\right|^{2 k}\right)^{\frac{1}{2 k}}+e^{-r t}\left(\mathbb{E}\left(\sum_{j=1}^{d}\left|\beta_{t}^{j}\right|^{2}\right)^{k}\right)^{\frac{1}{k}}\right] d t<+\infty, \quad 1 \leq j \leq d$, and $r>2 c_{2 k-1}$.

Then it holds

$$
\sup _{t \in \mathbb{R}_{+}} e^{-r k t} \mathbb{E}\left|Y_{t}\right|^{2 k} \leq K\left[\mathbb{E}\left|y_{0}\right|^{2 k}+\left(\int_{0}^{\infty} e^{-\frac{r}{2} t}\left(\mathbb{E}\left|\alpha_{t}\right|^{2 k}\right)^{\frac{1}{2 k}} d t\right)^{2 k}+\left(\int_{0}^{\infty} e^{-r t}\left(\mathbb{E}\left(\sum_{j=1}^{d}\left|\beta_{t}^{j}\right|^{2}\right)^{k}\right)^{\frac{1}{k}} d t\right)^{k}\right]
$$

where $K=K(\delta)$, for some appropriate $\delta>0$ and $r>2 c_{2 k-1}$.

Proof. The proof will be given for all $B^{j}, \beta^{j}$ 's bounded. Then the stochastic integral in the proof is a true (centered) martingale. The proof for the unbounded case follows immediately by standard localization and the Fatou lemma. 
Let $2 k, k \geq 2$ and apply the Itô formula to $e^{-r t}\left|Y_{t}\right|^{2 k}$ on $[0, t]$. The case $k \in[1 / 2,2)$ follows easily by the Hölder inequality.

$$
\begin{aligned}
\mathbb{E}\left(e^{-r k t}\left|Y_{t}\right|^{2 k}\right)= & \mathbb{E}\left|y_{0}\right|^{2 k}+2 k \mathbb{E} \int_{0}^{t} e^{-r k s}\left|Y_{s}\right|^{2 k-2}\left\langle A_{s} Y_{s}+\alpha_{s}, Y_{s}\right\rangle d s \\
& +2 k(k-1) \sum_{j=1}^{d} \mathbb{E} \int_{0}^{t} e^{-r k s}\left|Y_{s}\right|^{2 k-4}\left\langle B_{s}^{j} Y_{s}+\beta_{s}^{j}, Y_{s}\right\rangle^{2} d s \\
& +k \sum_{j=1}^{d} \mathbb{E} \int_{0}^{t} e^{-r k s}\left|Y_{s}\right|^{2 k-2}\left\langle B_{s}^{j} Y_{s}+\beta_{s}^{j}, B_{s}^{j} Y_{s}+\beta_{s}^{j}\right\rangle d s \\
& -r k \mathbb{E} \int_{0}^{t} e^{-r k s}\left|Y_{s}\right|^{2 k} d s \\
\leq & \mathbb{E}\left|y_{0}\right|^{2 k}+2 k \mathbb{E} \int_{0}^{t} e^{-r k s}\left|Y_{s}\right|^{2 k-2}\left[\left\langle A_{s} Y_{s}, Y_{s}\right\rangle+(2 k-1) \sum_{j=1}^{d}\left|B^{j} Y_{s}\right|^{2}\right] d s \\
& +2 k \mathbb{E} \int_{0}^{t} e^{-r k s}\left|Y_{s}\right|^{2 k-1}\left|\alpha_{s}\right| d s+2 k(2 k-1) \mathbb{E} \int_{0}^{t} e^{-r k s}\left|Y_{s}\right|^{2 k-2}\left(\sum_{j=1}^{d}\left|\beta_{s}^{j}\right|^{2}\right) d s \\
& -r k \mathbb{E} \int_{0}^{t} e^{-r k s}\left|Y_{s}\right|^{2 k} d s .
\end{aligned}
$$

Now, using Hölder and the weighted Young inequality $a b \leq \frac{a^{p} \delta^{p}}{p}+\frac{b^{q}}{q \delta^{q}}, a, b \geq 0, \delta>0$, the remaining terms can be treated as follows

$$
\begin{aligned}
& 2 k \mathbb{E} \int_{0}^{t} e^{-r k s}\left|Y_{s}\right|^{2 k-1}\left|\alpha_{s}\right| d s \leq 2 k\left(\sup _{t \in \mathbb{R}_{+}} e^{-r k t} \mathbb{E}\left(\left|Y_{t}\right|^{2 k-1}\right)^{\frac{2 k}{2 k-1}}\right)^{\frac{2 k-1}{2 k}} \int_{0}^{\infty} e^{-\frac{r}{2} t}\left(\mathbb{E}\left|\alpha_{t}\right|^{2 k}\right)^{\frac{1}{2 k}} d t \\
& \leq(2 k-1) \delta^{\frac{2 k}{2 k-1}}\left(\sup _{t \in \mathbb{R}_{+}} e^{-r k t} \mathbb{E}\left|Y_{t}\right|^{2 k}\right)+\frac{1}{\delta^{2 k}}\left(\int_{0}^{\infty} e^{-\frac{r}{2} t}\left(\mathbb{E}\left|\alpha_{t}\right|^{2 k}\right)^{\frac{1}{2 k}} d t\right)^{2 k}
\end{aligned}
$$

and similarly

$$
\begin{aligned}
& 2 k(2 k-1) \mathbb{E} \int_{0}^{t} e^{-r k s}\left|Y_{s}\right|^{2 k-2}\left(\sum_{j=1}^{d}\left|\beta_{s}^{j}\right|^{2}\right) d s \leq 2(k-1)(2 k-1) \delta \frac{2 k}{2 k-2}\left(\sup _{t \in \mathbb{R}_{+}} e^{-r k t} \mathbb{E}\left|Y_{t}\right|^{2 k}\right) \\
& \quad+2(2 k-1) \frac{1}{\delta^{k}}\left(\int_{0}^{\infty} e^{-r t}\left(\mathbb{E} \sum_{j=1}^{d}\left|\beta_{t}^{j}\right|^{2 k}\right)^{\frac{1}{k}} d t\right)^{k} .
\end{aligned}
$$

The estimate (29) easily follows by substituting (31) and (32) into (30), by taking $\sup _{t>0}$ on both sides and finally by choosing $\delta>0$ such that $1-(2 k-1) \delta^{\frac{2 k}{2 k-1}}-2(k-1)(2 k-1) \delta^{\frac{2 k}{2 k-2}}>0$ and $r>2 c_{2 k-1}$.

Before proceeding, let us recall that by $\bar{X}$ and $X^{\varepsilon}$ we mean the solution to (5) in the space $L_{\mathcal{F}}^{2,-r}\left(\mathbb{R}_{+} ; \mathbb{R}^{n}\right)$, for $r>2 c_{1 / 2}$, corresponding to $\bar{u}(\cdot)$ and $u^{\varepsilon}(\cdot)$, respectively. $y^{\varepsilon}$ and $z^{\varepsilon}$ are the solutions to (27) and (28), respectively.

Proposition 1. Suppose Hypotheses (H1)-(H4) hold and $r>2 c_{1 / 2}$. Define $\xi_{t}^{\varepsilon}:=X_{t}^{\varepsilon}-\bar{X}_{t}, \eta_{t}^{\varepsilon}:=$ $\xi_{t}^{\varepsilon}-y_{t}^{\varepsilon}$ and $\zeta_{t}^{\varepsilon}:=\xi_{t}^{\varepsilon}-y_{t}^{\varepsilon}-z_{t}^{\varepsilon}, t \geq 0$. Then there exist $\rho_{1}, \ldots, \rho_{5}>0$ such that for $k=1,2, \ldots$ it holds

(i) $\sup _{t \in \mathbb{R}_{+}} e^{-\rho_{1} k t} \mathbb{E}\left|\xi_{t}^{\varepsilon}\right|^{2 k}=O\left(\varepsilon^{k}\right)$,

(ii) $\sup _{t \in \mathbb{R}_{+}} e^{-\rho_{2} k t} \mathbb{E}\left|y_{t}^{\varepsilon}\right|^{2 k}=O\left(\varepsilon^{k}\right)$,

(iii) $\sup _{t \in \mathbb{R}_{+}} e^{-\rho_{3} k t} \mathbb{E}\left|z_{t}^{\varepsilon}\right|^{2 k}=O\left(\varepsilon^{2 k}\right)$,

(iv) $\sup _{t \in \mathbb{R}_{+}} e^{-\rho_{4} k t} \mathbb{E}\left|\eta_{t}^{\varepsilon}\right|^{2 k}=O\left(\varepsilon^{2 k}\right)$,

(v) $\sup _{t \in \mathbb{R}_{+}} e^{-\rho_{5} k t} \mathbb{E}\left|\zeta_{t}^{\varepsilon}\right|^{2 k}=o\left(\varepsilon^{2 k}\right)$. 
Proof. See Appendix.

Before giving a preliminary expansion of the cost, we state the following

Lemma 2. If $g \in C^{2}\left(\mathbb{R}^{n} ; \mathbb{R}\right)$ then the following equality holds for every $x, \bar{x} \in \mathbb{R}^{n}$

$$
g(x)=g(\bar{x})+\left\langle D_{x} g(\bar{x}), x-\bar{x}\right\rangle+\int_{0}^{1}\left\langle\theta D_{x}^{2} g(\theta \bar{x}+(1-\theta)(x-\bar{x})), x-\bar{x}\right\rangle d \theta .
$$

Proposition 2. The following expansion holds for the cost functional

$$
\begin{aligned}
J\left(u^{\varepsilon}(\cdot)\right)-J(\bar{u}(\cdot))= & \mathbb{E} \int_{0}^{\infty} e^{-r t}\left\langle D_{x} f\left(\bar{X}_{t}, \bar{u}_{t}\right), y_{t}^{\varepsilon}+z_{t}^{\varepsilon}\right\rangle d t \\
& +\mathbb{E} \int_{0}^{\infty} e^{-r t}\left[\frac{1}{2}\left\langle D_{x}^{2} f\left(\bar{X}_{t}, \bar{u}_{t}\right) y_{t}^{\varepsilon}, y_{t}^{\varepsilon}\right\rangle+\delta f\left(\bar{X}_{t}, \bar{u}_{t}\right)\right] d t+o(\varepsilon),
\end{aligned}
$$

where the discount factor $r \geq \max _{i=1, \ldots, 5}\left\{\rho_{i}\right\}$ and $\rho_{i}$ are the individual discount factors from Proposition 1 .

Proof of Proposition 2. Thanks to Lemma 2, we have

$$
\begin{aligned}
J\left(u^{\varepsilon}(\cdot)\right)-J(\bar{u}(\cdot))= & \mathbb{E} \int_{0}^{\infty} e^{-r t}\left[f\left(X_{t}^{\varepsilon}, u_{t}^{\varepsilon}\right)-f\left(\bar{X}_{t}, \bar{u}_{t}\right)\right] d t \\
= & \mathbb{E} \int_{0}^{\infty} e^{-r t}\left[\delta f\left(\bar{X}_{t}, \bar{u}_{t}\right)+\left\langle D_{x} f\left(\bar{X}_{t}, u_{t}^{\varepsilon}\right), \xi_{t}^{\varepsilon}\right\rangle\right] d t \\
& +\mathbb{E} \int_{0}^{\infty} e^{-r t} \int_{0}^{1}\left\langle\theta D_{x}^{2} f\left(\theta \bar{X}_{t}+(1-\theta) X_{t}^{\varepsilon}, u_{t}^{\varepsilon}\right) \xi_{t}^{\varepsilon}, \xi_{t}^{\varepsilon}\right\rangle d \theta d t .
\end{aligned}
$$

Finally, Proposition 1 gives

$$
\begin{aligned}
J\left(u^{\varepsilon}(\cdot)\right)-J(\bar{u}(\cdot))=\mathbb{E} & \int_{0}^{\infty} e^{-r t}\left[\delta f\left(\bar{X}_{t}, \bar{u}_{t}\right)+\left\langle\delta D_{x} f\left(\bar{X}_{t}, \bar{u}_{t}\right), \xi_{t}^{\varepsilon}\right\rangle\right. \\
& +\left\langle D_{x} f\left(\bar{X}_{t}, \bar{u}_{t}\right), y_{t}^{\varepsilon}+z_{t}^{\varepsilon}\right\rangle+\left\langle D_{x} f\left(\bar{X}_{t}, \bar{u}_{t}\right), \zeta_{t}^{\varepsilon}\right\rangle \\
& +\int_{0}^{1}\left\langle\theta\left(D_{x}^{2} f\left(\theta \bar{X}_{t}+(1-\theta) X_{t}^{\varepsilon}, u_{t}^{\varepsilon}\right)-D_{x}^{2} f\left(\bar{X}_{t}, u_{t}^{\varepsilon}\right)\right) \xi_{t}^{\varepsilon}, \xi_{t}^{\varepsilon}\right\rangle d \theta \\
& +\frac{1}{2}\left\langle\delta D_{x}^{2} f\left(\bar{X}_{t}, \bar{u}_{t}\right) \xi_{t}^{\varepsilon}, \xi_{t}^{\varepsilon}\right\rangle+\frac{1}{2}\left\langle D_{x}^{2} f\left(\bar{X}_{t}, \bar{u}_{t}\right) y_{t}^{\varepsilon}, y_{t}^{\varepsilon}\right\rangle \\
& \left.+\frac{1}{2}\left\langle D_{x}^{2} f\left(\bar{X}_{t}, \bar{u}_{t}\right) \eta_{t}^{\varepsilon}, \xi_{t}^{\varepsilon}+y_{t}^{\varepsilon}\right\rangle\right] d t \\
= & \mathbb{E} \int_{0}^{\infty} e^{-r t}\left\langle D_{x} f\left(\bar{X}_{t}, \bar{u}_{t}\right), y_{t}^{\varepsilon}+z_{t}^{\varepsilon}\right\rangle d t \\
+ & \mathbb{E} \int_{0}^{\infty} e^{-r t}\left[\frac{1}{2}\left\langle D_{x}^{2} f\left(\bar{X}_{t}, \bar{u}_{t}\right) y_{t}^{\varepsilon}, y_{t}^{\varepsilon}\right\rangle+\delta f\left(\bar{X}_{t}, \bar{u}_{t}\right)\right] d t+o(\varepsilon),
\end{aligned}
$$

which completes the proof.

\section{First adjoint EQUation}

The first adjoint process naturally arises as a solution to an appropriate BSDE whose driver can be obtained by differentiating the Hamiltonian function with respect to the state variable $x$. In some sense, the first adjoint process is dual to the linearized state equation (27) and it can have the interpretation of generalized (in the sense of time-dependent and stochastic) Lagrange multipliers. In the classical setting for BSDEs, the terminal condition is given a priori. On the contrary, here the BSDE is solved on infinite time horizon and the behaviour at infinity is not known. Yet the existence and uniqueness result can be derived for processes being in some exponentially-weighted $L^{2}$ space.

In our case, the first order adjoint equation on infinite time horizon has the following form

$$
d p_{t}=-\left[D_{x} b\left(\bar{X}_{t}, \bar{u}_{t}\right)^{T} p_{t}+D_{x} \sigma\left(\bar{X}_{t}, \bar{u}_{t}\right)^{T} q_{t}-D_{x} f\left(\bar{X}_{t}, \bar{u}_{t}\right)-r p_{t}\right] d t+q_{t} d W_{t}
$$


where $D_{x} \sigma\left(\bar{X}_{t}, \bar{u}_{t}\right)^{T} q_{t}:=\sum_{j=1}^{d} D_{x} \sigma^{j}\left(\bar{X}_{t}, \bar{u}_{t}\right)^{T} q_{t}^{j} \in \mathbb{R}^{n}$ and $\left(\bar{X}_{t}, \bar{u}_{t}\right)$ is an optimal pair.

Let us start the analysis by proving an a priori estimate for the difference of solutions to (35). To do so, the following estimate will be of a particular interest since it allows to transfer the joint monotonicity property to the BSDE

$$
\begin{aligned}
\left\langle D_{x} \sigma\left(\bar{X}_{t}, \bar{u}_{t}\right)^{T} q_{t}, p_{t}\right\rangle & =\sum_{j=1}^{d}\left\langle D_{x} \sigma^{j}\left(\bar{X}_{t}, \bar{u}_{t}\right)^{T} q_{t}^{j}, p_{t}\right\rangle=\sum_{j=1}^{d}\left\langle q_{t}^{j}, D_{x} \sigma^{j}\left(\bar{X}_{t}, \bar{u}_{t}\right) p_{t}\right\rangle \\
\leq \frac{1}{2} \sum_{j=1}^{d}\left|q_{t}^{j}\right|^{2}+\frac{1}{2} \sum_{j=1}^{d}\left|D_{x} \sigma^{j}\left(\bar{X}_{t}, \bar{u}_{t}\right) p_{t}\right|^{2} & \leq \frac{1}{2}\left\|q_{t}\right\|^{2}+\frac{1}{2}\left\|D_{x} \sigma\left(\bar{X}_{t}, \bar{u}_{t}\right) p_{t}\right\|_{2}^{2} .
\end{aligned}
$$

Lemma 3. Let $\left(p^{1}, q^{1}\right)$ and $\left(p^{2}, q^{2}\right)$ be two solutions to BSDE (35) belonging to the space $L_{\mathcal{F}}^{2,-r}\left(\mathbb{R}_{+} ; \mathbb{R}^{n} \times\right.$ $\left.\mathbb{R}^{n \times d}\right)$, for $r>2 c_{1 / 2}$, corresponding to $f=f^{1}$ and $f=f^{2}$. Then the following estimate holds true

$$
\begin{aligned}
\mathbb{E} \int_{0}^{\infty} e^{-r t} & \left(\left(r-2 c_{1 / 2}-\delta\right)\left|p_{t}^{1}-p_{t}^{2}\right|^{2}+\frac{1}{2}|| q_{t}^{1}-q_{t}^{2} \|^{2}\right) d t \\
\leq & \frac{1}{\delta} \mathbb{E} \int_{0}^{\infty} e^{-r t}\left|D_{x} f^{1}\left(\bar{X}_{t}, \bar{u}_{t}\right)-D_{x} f^{2}\left(\bar{X}_{t}, \bar{u}_{t}\right)\right|^{2} d t
\end{aligned}
$$

where $\delta>0$ is sufficiently small.

Proof. Applying Itô formula to $e^{-r t}\left|p_{t}^{1}-p_{t}^{2}\right|^{2}$ gives

$$
\begin{aligned}
\mid p_{0}^{1}- & \left.p_{0}^{2}\right|^{2}+\mathbb{E} \int_{0}^{\infty} e^{-r t}\left(-r\left|p_{t}^{1}-p_{t}^{2}\right|^{2}+\left\|q_{t}^{1}-q_{t}^{2}\right\|^{2}\right) d t \\
= & \mathbb{E} \int_{0}^{\infty} 2 e^{-r t}\left\langle p_{t}^{1}-p_{t}^{2}, D_{x} b\left(\bar{X}_{t}, \bar{u}_{t}\right)^{T}\left(p_{t}^{1}-p_{t}^{2}\right)+D_{x} \sigma\left(\bar{X}_{t}, \bar{u}_{t}\right)^{T}\left(q_{t}^{1}-q_{t}^{2}\right)\right\rangle d t \\
& +\mathbb{E} \int_{0}^{\infty} 2 e^{-r t}\left\langle p_{t}^{1}-p_{t}^{2},-r\left(p_{t}^{1}-p_{t}^{2}\right)+D_{x} f^{1}\left(\bar{X}_{t}, \bar{u}_{t}\right)-D_{x} f^{2}\left(\bar{X}_{t}, \bar{u}_{t}\right)\right\rangle d t \\
\leq & \mathbb{E} \int_{0}^{\infty} 2 e^{-r t}\left(\left\langle p_{t}^{1}-p_{t}^{2}, D_{x} b\left(\bar{X}_{t}, \bar{u}_{t}\right)^{T}\left(p_{t}^{1}-p_{t}^{2}\right)\right\rangle+\frac{1}{2}\left\|D_{x} \sigma\left(\bar{X}_{t}, \bar{u}_{t}\right)\left(p_{t}^{1}-p_{t}^{2}\right)\right\|_{2}^{2}\right) d t \\
& +\mathbb{E} \int_{0}^{\infty} 2 e^{-r t}\left(\frac{1}{2}|| q_{t}^{1}-q_{t}^{2} \|^{2}-r\left|p_{t}^{1}-p_{t}^{2}\right|^{2}\right) d t \\
& +\mathbb{E} \int_{0}^{\infty} 2 e^{-r t}\left(\frac{\delta}{2}\left|p_{t}^{1}-p_{t}^{2}\right|^{2}+\frac{1}{2 \delta}\left|D_{x} f^{1}\left(\bar{X}_{t}, \bar{u}_{t}\right)-D_{x} f^{2}\left(\bar{X}_{t}, \bar{u}_{t}\right)\right|^{2}\right) d t \\
\leq & \mathbb{E} \int_{0}^{\infty} e^{-r t}\left[\left(2 c_{1 / 2}-2 r+\delta\right)\left|p_{t}^{1}-p_{t}^{2}\right|^{2}+\frac{1}{2}\left\|q_{t}^{1}-q_{t}^{2}\right\|^{2}\right] d t \\
& +\mathbb{E} \int_{0}^{\infty} e^{-r t} \frac{1}{\delta}\left|D_{x} f^{1}\left(\bar{X}_{t}, \bar{u}_{t}\right)-D_{x} f^{2}\left(\bar{X}_{t}, \bar{u}_{t}\right)\right|^{2} d t
\end{aligned}
$$

where we have used the estimate (36), joint monotonicity assumption (H4) and weighted Young inequality. The conclusion easily follows.

Before giving the proof of existence and uniqueness for the first adjoint equation we produce a preliminary result in finite time horizon $T>0$. Let us consider the following equation:

$$
\left\{\begin{array}{l}
d p_{t}=-\left[D_{x} b\left(\bar{X}_{t}, \bar{u}_{t}\right)^{T} p_{t}+D_{x} \sigma\left(\bar{X}_{t}, \bar{u}_{t}\right)^{T} q_{t}-D_{x} f\left(\bar{X}_{t}, \bar{u}_{t}\right)-r p_{t}\right] d t+q_{t} d W_{t}, \\
p_{T}=0,
\end{array}\right.
$$

where $T>0$ is arbitrary but fixed.

As far as we know, no results in the literature can be used to solve this equation due to the polynomial growth of $D_{x} \sigma\left(\bar{X}_{t}, \bar{u}_{t}\right)^{T}$ in front of $q_{t}$. In order to produce existence of a solution to such equation we exploit some duality arguments.

Theorem 3. Under Hypotheses (H1)-(H5) equation (37) admits a unique solution $(p, q)$ which belongs to $\in L_{\mathcal{F}}^{2}\left([0, T] ; \mathbb{R}^{n}\right) \times L_{\mathcal{F}}^{2}\left([0, T] ; \mathbb{R}^{n \times d}\right)$, for each $T>0$. 
Proof. The proof consists in three steps. First, the diffusion term $\sigma$ is approximated so that there exists a solution for each approximating backward equation, by classical results. Second, a duality between these approximate solutions and a properly perturbed first variation equation is established. The last step consists in constructing a unique solution to the original equation (on a finite horizon) by some compactness arguments.

Let us define a sequence of Lipschitz-continuous maps $\sigma^{n}$ with $\sigma^{n}(x) \rightarrow \sigma(x)$ as $n \rightarrow \infty$, for all $x \in \mathbb{R}^{n}$ so that the joint monotonicity property still holds. An example of such approximation can be given by (see [3] )

$$
\sigma^{n}(x)= \begin{cases}\sigma(x), & \text { if }|x| \leq n, \\ \sigma\left(\frac{(n+1) x}{|x|}\right), & \text { if }|x| \geq n+1 .\end{cases}
$$

Then, for each $n$, the following approximating equation

$$
\left\{\begin{array}{l}
d p_{t}^{n}=-\left[D_{x} b\left(\bar{X}_{t}, \bar{u}_{t}\right)^{T} p_{t}^{n}+D_{x} \sigma^{n}\left(\bar{X}_{t}, \bar{u}_{t}\right)^{T} q_{t}^{n}-D_{x} f\left(\bar{X}_{t}, \bar{u}_{t}\right)-r p_{t}^{n}\right] d t+q_{t}^{n} d W_{t}, \\
p_{T}^{n}=0,
\end{array}\right.
$$

admits a unique solution $\left(p_{t}^{n}, q_{t}^{n}\right) \in L_{\mathcal{F}}^{2}\left([0, T] ; \mathbb{R}^{n}\right) \times L_{\mathcal{F}}^{2}\left([0, T] ; \mathbb{R}^{n \times d}\right)$ thanks to Briand et al. [2], Theorem 4.1.

Now, to establish the duality, consider for all $n \in \mathbb{N}$ and for all $\gamma(\cdot) \in L_{\mathcal{F}}^{2}\left([0, T] ; \mathbb{R}^{n}\right)$ and $\eta(\cdot) \in$ $L_{\mathcal{F}}^{2}\left([0, T] ; \mathbb{R}^{n \times d}\right)$ the following perturbed first variation equation

$$
\left\{\begin{array}{l}
d y_{t}^{n}=\left(D_{x} b\left(\bar{X}_{t}, \bar{u}_{t}\right) y_{t}^{n}-r y_{t}^{n}+\gamma_{t}\right) d t+\left(D_{x} \sigma^{n}\left(\bar{X}_{t}, \bar{u}_{t}\right) y_{t}^{n}+\eta_{t}\right) d W_{t}, t \in(0, T], \\
y_{0}^{n}=0 .
\end{array}\right.
$$

Due to Theorem 2 we know that the above equation has a unique solution in $L_{\mathcal{F}}^{2}\left([0, T] ; \mathbb{R}^{n}\right)$ for each $n$. Moreover, using dissipativity it is easy to show that there exists $K>0$ not depending on $n$ such that

$$
\mathbb{E} \int_{0}^{T}\left|y_{t}^{n}\right|^{2} d t \leq K\left[\mathbb{E} \int_{0}^{T}\left|\gamma_{t}\right|^{2} d t+\mathbb{E} \int_{0}^{T}\left\|\eta_{t}\right\|^{2} d t\right] .
$$

Next, by applying the Itô formula to $d\left\langle y_{t}^{n}, p_{t}^{n}\right\rangle$ we establish the duality relation

$$
\mathbb{E} \int_{0}^{T}\left\langle p_{t}^{n}, \gamma_{t}\right\rangle d t+\mathbb{E} \int_{0}^{T} \operatorname{Tr}\left\{q_{t}^{n}\left(\eta_{t}\right)^{T}\right\} d t=-\mathbb{E} \int_{0}^{T}\left\langle D_{x} f\left(\bar{X}_{t}, \bar{u}_{t}\right), y_{t}^{n}\right\rangle d t .
$$

Let us define the set $\mathcal{A}:=\left\{\gamma(\cdot) \in L_{\mathcal{F}}^{2}\left([0, T] ; \mathbb{R}^{n}\right):\|\gamma\|_{L_{\mathcal{F}}^{2}\left([0, T] ; \mathbb{R}^{n}\right)} \leq 1\right\}$. If we take $\eta \equiv 0$ in (41) we get

$$
\begin{aligned}
\left(\mathbb{E} \int_{0}^{T}\left|p_{t}^{n}\right|^{2} d t\right)^{1 / 2} & \leq \sup _{\gamma \in \mathcal{A}}\left[\mathbb{E} \int_{0}^{T}\left|\left\langle D_{x} f\left(\bar{X}_{t}, \bar{u}_{t}\right), y_{t}^{n}\right\rangle\right| d t\right] \\
& \leq \sup _{\gamma \in \mathcal{A}}\left[\left(\mathbb{E} \int_{0}^{T}\left|D_{x} f\left(\bar{X}_{t}, \bar{u}_{t}\right)\right|^{2} d t\right)^{1 / 2}\left(\mathbb{E} \int_{0}^{T}\left|y_{t}^{n}\right|^{2} d t\right)^{1 / 2}\right] \\
& \leq C\left(\mathbb{E} \int_{0}^{T}\left|D_{x} f\left(\bar{X}_{t}, \bar{u}_{t}\right)\right|^{2} d t\right)^{1 / 2} .
\end{aligned}
$$

If we repeat the same argument with $\gamma \equiv 0$, instead of $\eta$, we finally get

$$
\left\|p_{t}^{n}\right\|_{L_{\mathcal{F}}^{2}\left([0, T] ; \mathbb{R}^{n}\right)}^{2}+\left\|q_{t}^{n}\right\|_{L_{\mathcal{F}}^{2}\left([0, T] ; \mathbb{R}^{n \times d}\right)}^{2} \leq C \mathbb{E} \int_{0}^{T}\left|D_{x} f\left(\bar{X}_{t}, \bar{u}_{t}\right)\right|^{2} d t .
$$

This way we have obtained a uniform estimate (with respect to $n$ ) of the $L_{\mathcal{F}}^{2}$-norm of $\left(p^{n}, q^{n}\right)$. Hence there exists a subsequence, denoted by abuse of notation again as $\left(p^{n}, q^{n}\right)$, which converges weakly in $L_{\mathcal{F}}^{2}\left([0, T] ; \mathbb{R}^{n}\right) \times L_{\mathcal{F}}^{2}\left([0, T] ; \mathbb{R}^{n \times d}\right)$ to a couple $(p, q)$. Our goal is to verify that $(p, q)$ is the solution to the limit equation

$$
\left\{\begin{array}{l}
d p_{t}=-\left[D_{x} b\left(\bar{X}_{t}, \bar{u}_{t}\right)^{T} p_{t}+D_{x} \sigma\left(\bar{X}_{t}, \bar{u}_{t}\right)^{T} q_{t}-D_{x} f\left(\bar{X}_{t}, \bar{u}_{t}\right)-r p_{t}\right] d t+q_{t} d W_{t}, \\
p_{T}=0 .
\end{array}\right.
$$


To do so, we note that due to the linearity of the equation, it is enough to prove that each term of the approximating equation weakly converges to the corresponding term in the limit equation. Let us start by studying the term

$$
\begin{aligned}
\left\langle D_{x} \sigma^{n}\left(\bar{X}_{t}, \bar{u}_{t}\right)^{T} q_{t}^{n}, v\right\rangle & =\left\langle q_{t}^{n}, D_{x} \sigma^{n}\left(\bar{X}_{t}, \bar{u}_{t}\right) v\right\rangle \\
& =\left\langle q_{t}^{n}, D_{x} \sigma\left(\bar{X}_{t}, \bar{u}_{t}\right) v\right\rangle+\left\langle q_{t}^{n}, D_{x} \sigma^{n}\left(\bar{X}_{t}, \bar{u}_{t}\right) v-D_{x} \sigma^{n}\left(\bar{X}_{t}, \bar{u}_{t}\right) v\right\rangle .
\end{aligned}
$$

As $n \rightarrow \infty$, the right hand side converges to

$$
\left\langle q_{t}, D_{x} \sigma\left(\bar{X}_{t}, \bar{u}_{t}\right) v\right\rangle=\left\langle D_{x} \sigma\left(\bar{X}_{t}, \bar{u}_{t}\right)^{T} q_{t}, v\right\rangle,
$$

thanks to the pointwise convergence of the derivative of $\sigma^{n}$. Indeed, $D_{x} \sigma^{n}(x)=D_{x} \sigma(x)$, if $|x| \leq n$, and the derivative is bounded. Regarding the noise term, let us notice that the map $q \rightarrow \int_{0}^{T} q_{t} d W_{t}$ is linear and continuous, hence weakly continuous. The other terms are easy to treat.

For the uniqueness part it is enough to use a version of Lemma 3 on finite time horizon. Then we have existence and uniqueness of a solution in finite time horizon and the proof is finished.

\section{Remark 3.}

(a) The introduction of the term $-r y_{t}^{n}$ in (39) is due to the choice of the scalar product used for establishing duality. If one considers a scalar product in $L^{2,-r}$ rather than in $L^{2}$ then the additional term $-r y_{t}^{n}$ can be omitted.

(b) An alternative approach to obtain the uniform estimate can be the one following Pardoux [16]. Indeed, applying the Itô formula gives

$$
\begin{aligned}
\mathbb{E}\left|p_{t}^{n}\right|^{2}= & 2 \mathbb{E} \int_{t}^{T}\left[\left\langle p_{s}^{n}, D_{x} b\left(\bar{X}_{s}, \bar{u}_{s}\right) p_{s}^{n}\right\rangle+\left\langle p_{s}^{n}, D_{x} \sigma^{n}\left(\bar{X}_{s}, \bar{u}_{t}\right)^{T} q_{s}^{n}\right\rangle+\left\langle p_{s}^{n}, D_{x} f\left(\bar{X}_{s}, \bar{u}_{s}\right)\right\rangle\right] d s \\
& -2 r \mathbb{E} \int_{t}^{T}\left|p_{s}^{n}\right|^{2} d s-\mathbb{E} \int_{t}^{T}\left\|q_{s}^{n}\right\|^{2} d s,
\end{aligned}
$$

which, thanks to the joint monotonicity of $b, \sigma^{n}$ and weighted Young inequality, produces

$$
\begin{aligned}
\mathbb{E}\left|p_{t}^{n}\right|^{2}+ & \left(2 r-\varepsilon-2 c_{1}\right) \mathbb{E} \int_{t}^{T}\left|p_{s}^{n}\right|^{2} d s+\left(1-\frac{1}{2}\right) \mathbb{E} \int_{t}^{T}\left\|q_{s}^{n}\right\|^{2} d s \\
\leq & \frac{1}{\varepsilon} \mathbb{E} \int_{t}^{T}\left|D_{x} f\left(\bar{X}_{s}, \bar{u}_{s}\right)\right|^{2} d s,
\end{aligned}
$$

for all $t \in[0, T], \varepsilon>0$ and $r>c_{1}$. Again, we have a uniform estimate (in $n$ ) for the left hand side and the relative compactness argument can be applied as before. Note that this approach gives another restriction on $r$ than the one used in the proof.

(c) All the results of this section can be made more general when considering general weighted Young inequality $a b \leq \frac{p}{2} a^{2}+\frac{1}{2 p} b^{2}, p>0$ in (36) rather than the usual Young inequality with $p=1$.

Now we are ready for the following

Theorem 4. Under Hypotheses $(H 1)-(H 5)$, there exists $r>0$ such that equation (35) admits a unique solution $(p, q)$ which belongs to $L_{\mathcal{F}}^{2,-r}\left(\mathbb{R}_{+} ; \mathbb{R}^{n}\right) \times L_{\mathcal{F}}^{2,-r}\left(\mathbb{R}_{+} ; \mathbb{R}^{n \times d}\right)$.

Proof. Following Peng and Shi [18], Theorem 4, define for all $k \in \mathbb{N}$

$$
\varphi_{t}^{k}:=D_{x} f\left(\bar{X}_{t}, \bar{u}_{t}\right) \chi_{[0, k]}(t), \quad t \in \mathbb{R}_{+},
$$

which converges to $D_{x} f\left(\bar{X}_{t}, \bar{u}_{t}\right)$ as $k \rightarrow \infty$. We define the solution to the following approximate equation on infinite time horizon

$$
d p_{t}^{k}=-\left[D_{x} b\left(\bar{X}_{t}, \bar{u}_{t}\right)^{T} p_{t}^{k}+D_{x} \sigma\left(\bar{X}_{t}, \bar{u}_{t}\right)^{T} q_{t}^{k}-\varphi_{t}^{k}-r p_{t}^{k}\right] d t+q_{t}^{k} d W_{t}
$$

as a process solving the following BSDE on a finite time horizon

$$
\left\{\begin{array}{l}
d p_{t}^{k}=-\left[D_{x} b\left(\bar{X}_{t}, \bar{u}_{t}\right)^{T} p_{t}^{k}+D_{x} \sigma\left(\bar{X}_{t}, \bar{u}_{t}\right)^{T} q_{t}^{k}-\varphi_{t}^{k}-r p_{t}^{k}\right] d t+q_{t}^{k} d W_{t}, \\
p_{k}^{k}=0
\end{array}\right.
$$


and which is identically zero for $t \in(k, \infty)$. Such solution exists for each $k$ due to Theorem 3 , Using the a priori estimate given in Lemma 3 , it is easy to see that there exists $r$ such that the sequence of solutions $\left(p_{t}^{k}, q_{t}^{k}\right)$ forms a Cauchy sequence in $L_{\mathcal{F}}^{2,-r}\left(\mathbb{R}_{+} ; \mathbb{R}^{n}\right) \times L_{\mathcal{F}}^{2,-r}\left(\mathbb{R}_{+} ; \mathbb{R}^{n \times d}\right)$ and that the limiting processes $\left(p_{t}, q_{t}\right)$ solves (35). Uniqueness is straightforward due to Lemma 3 ,

\section{SECOND ADJOINT}

The second adjoint equation has the following form

$$
\begin{aligned}
-d P(t)=[ & D_{x} b\left(\bar{X}_{t}, \bar{u}_{t}\right)^{T} P_{t}+P_{t} D_{x} b\left(\bar{X}_{t}, \bar{u}_{t}\right) \\
& +\sum_{j=1}^{d} D_{x} \sigma^{j}\left(\bar{X}_{t}, \bar{u}_{t}\right)^{T} P_{t} D_{x} \sigma^{j}\left(\bar{X}_{t}, \bar{u}_{t}\right) \\
& +\sum_{j=1}^{d}\left(D_{x} \sigma^{j}\left(\bar{X}_{t}, \bar{u}_{t}\right)^{T} Q_{t}^{j}+Q_{t}^{j} D_{x} \sigma^{j}\left(\bar{X}_{t}, \bar{u}_{t}\right)\right) \\
& \left.+D_{x}^{2} H\left(\bar{X}_{t}, \bar{u}_{t}, p_{t}, q_{t}\right)-r_{t} P_{t}\right] d t-\sum_{j=1}^{d} Q_{t}^{j} d W_{t}^{j} .
\end{aligned}
$$

For a detailed discussion of the role of this equation see e.g. [24]. We can see that the term $\sum_{j=1}^{d} D_{x} \sigma^{j}\left(\bar{X}_{t}, \bar{u}_{t}\right)^{T} P_{t} D_{x} \sigma^{j}\left(\bar{X}_{t}, \bar{u}_{t}\right)$ destroys the dissipative behaviour of the dynamics in the sense that, in general

$$
\left\langle\sum_{j=1}^{d} D_{x} \sigma^{j}\left(\bar{X}_{t}, \bar{u}_{t}\right)^{T} P_{t} D_{x} \sigma^{j}\left(\bar{X}_{t}, \bar{u}_{t}\right), P_{t}\right\rangle_{2} \not \leq c_{p}\left\|P_{t}\right\|_{2}^{2} .
$$

Nontheless, see Remark 4 for one particular case. The lack of dissipativity prevents us from finding an a priori estimate of the solution. Hence the argument we adopted to solve the first adjoint is no longer helpful. The only information that can be useful to study the process $P_{t}$ comes from the first variation equation (27). It can be shown that $P_{t}$ is dual (in some sense explained later) to the process $Y_{t}^{\varepsilon}$ defined as $Y_{t}^{\varepsilon}=y_{t}^{\varepsilon}\left(y_{t}^{\varepsilon}\right)^{T}$. It is not difficult to verify that $Y_{t}^{\varepsilon}$ is a symmetric and positive (semi)definite matrix process. By using Itô formula it can be also shown that it is a solution to the following (matrix-valued) SDE

$$
\begin{aligned}
d Y_{t}^{\varepsilon}=[ & D_{x} b\left(\bar{X}_{t}, \bar{u}_{t}\right) Y_{t}^{\varepsilon}+Y_{t}^{\varepsilon} D_{x} b\left(\bar{X}_{t}, \bar{u}_{t}\right)^{T} \\
& \left.+\sum_{j=1}^{d} D_{x} \sigma^{j}\left(\bar{X}_{t}, \bar{u}_{t}\right) Y_{t}^{\varepsilon} D_{x} \sigma^{j}\left(\bar{X}_{t}, \bar{u}_{t}\right)^{T}+\Gamma(t)\right] d t \\
& +\sum_{j=1}^{d}\left[D_{x} \sigma^{j}\left(\bar{X}_{t}, \bar{u}_{t}\right) Y_{t}^{\varepsilon}+Y_{t}^{\varepsilon} D_{x} \sigma^{j}\left(\bar{X}_{t}, \bar{u}_{t}\right)^{T}+\Lambda^{j}(t)\right] d W_{t}^{j}, \\
Y_{0}^{\varepsilon}=0, &
\end{aligned}
$$

where

$$
\begin{aligned}
\Gamma(t):= & \sum_{j=1}^{d} \delta \sigma^{j}\left(\bar{X}_{t}, \bar{u}_{t}\right)\left(\delta \sigma^{j}\left(\bar{X}_{t}, \bar{u}_{t}\right)\right)^{T}+\sum_{j=1}^{d} D_{x} \sigma^{j}\left(\bar{X}_{t}, \bar{u}_{t}\right) y_{t}^{\varepsilon}\left(\delta \sigma^{j}\left(\bar{X}_{t}, \bar{u}_{t}\right)\right)^{T} \\
& +\sum_{j=1}^{d} \delta \sigma^{j}\left(\bar{X}_{t}, \bar{u}_{t}\right)\left(y_{t}^{\varepsilon}\right)^{T} D_{x} \sigma^{j}\left(\bar{X}_{t}, \bar{u}_{t}\right)^{T}
\end{aligned}
$$

and

$$
\Lambda(t)=\sum_{j=1}^{d} \Lambda^{j}(t):=\sum_{j=1}^{d}\left[\delta \sigma^{j}\left(\bar{X}_{t}, \bar{u}_{t}\right)\left(y_{t}^{\varepsilon}\right)^{T}+y_{t}^{\varepsilon}\left(\delta \sigma^{j}\left(\bar{X}_{t}, \bar{u}_{t}\right)\right)^{T}\right] .
$$

We also have the following 
Proposition 3. Under Hypotheses (H1)-(H5), there exists $r \in \mathbb{R}$ such that equation (47) has a unique solution $Y^{\varepsilon} \in L_{\mathcal{F}}^{2,-r}\left(\mathbb{R}_{+} ; \mathbb{R}^{n \times n}\right)$ and the following holds

$$
\mathbb{E} \int_{0}^{\infty} e^{-r t}\left\|Y_{t}^{\varepsilon}\right\|_{2}^{2} d t \leq K \mathbb{E} \int_{0}^{\infty} e^{-r t}\left\|\Gamma_{t}\right\|_{2}^{2} d t+K \sum_{j=1}^{d} \mathbb{E} \int_{0}^{\infty} e^{-r t}\left\|\Lambda_{t}^{j}\right\|_{2}^{2} d t,
$$

for some $K>0$.

Proof. See Appendix.

The crucial point here is that Proposition 3 holds true if and only if $Y_{t}^{\varepsilon}$ is the solution to equation (47), i.e. for $\Gamma$ and $\Lambda$ given by (48) and (49). For general (nonsymmetric) forcing terms $\Gamma$ and $\Lambda^{j} \in L_{\mathcal{F}}^{2,-r}\left(\mathbb{R}_{+} ; \mathbb{R}^{n \times n}\right)$ the corresponding process $Y_{t}$ can not be decomposed anymore as $y_{t} y_{t}^{T}$ for some process $y_{t}$. Due to this fact, it is not possible to apply a classical duality argument (as in [22] or [24]) to extract some information for $P$ and the corresponding BSDE.

Remark 4. Note that in the case of $\sigma$ Lipschitz (thus $D_{x} \sigma$ bounded) it is quite easy to derive the dissipativity of $P$ in sense of (46). This particular case can be treated in the same way as in the section on first adjoint equation.

7.1. Construction of $P_{t}$. Here we propose a different way to construct the process $P$, following ideas of Fuhrman et al. [7]. More precisely, will show that there exists $r>0$ and a well defined matrix-valued process $P$ such that the following duality relation holds

$$
\begin{aligned}
\mathbb{E} \int_{0}^{\infty} e^{-r t} \operatorname{Tr}\left[D_{x}^{2} H\left(\bar{X}_{t}, \bar{u}_{t}, p_{t}, q_{t}\right) Y_{t}^{\varepsilon}\right] d t \\
\quad=\sum_{j=1}^{d} \mathbb{E} \int_{0}^{\infty} e^{-r t}\left\langle P_{t} \delta \sigma^{j}\left(\bar{X}_{t}, \bar{u}_{t}\right), \delta \sigma^{j}\left(\bar{X}_{t}, \bar{u}_{t}\right)\right\rangle d t+o(\varepsilon) .
\end{aligned}
$$

Once we have this relation, it is easy to prove the stochastic maximum principle using usual arguments. The strategy to do so will be the following.

Dual identity satisfied by $P$ : For $t \geq 0$ and an arbitrary vector $\gamma \in \mathbb{R}^{n}$, let us consider the following SDE

$$
\left\{\begin{aligned}
d y_{s}^{t, \gamma} & =D_{x} b\left(\bar{X}_{s}, \bar{u}_{s}\right) y_{s}^{t, \gamma} d s+\sum_{j=1}^{d} D_{x} \sigma^{j}\left(\bar{X}_{s}, \bar{u}_{s}\right) y_{s}^{t, \gamma} d W_{s}^{j}, s \geq t \\
y_{t}^{t, \gamma} & =\gamma
\end{aligned}\right.
$$

By repeating the arguments by Yong and Zhou 24], Chapter 3, the SDE for the product $y_{s}^{t, \eta}\left(y_{s}^{t, \gamma}\right)^{T}$ is of the form (with the notation $A_{t} \equiv D_{x} b\left(\bar{X}_{t}, \bar{u}_{t}\right)$ and $B_{t}^{j} \equiv D_{x} \sigma^{j}\left(\bar{X}_{t}, \bar{u}_{t}\right)$ )

$$
\begin{aligned}
d\left(y_{s}^{t, \eta}\left(y_{s}^{t, \gamma}\right)^{T}\right)= & {\left[A_{s} y_{s}^{t, \eta}\left(y_{s}^{t, \gamma}\right)^{T}+y_{s}^{t, \eta}\left(y_{s}^{t, \gamma}\right)^{T} A_{s}^{T}+\sum_{j=1}^{d} B_{s}^{j} y_{s}^{t, \eta}\left(y_{s}^{t, \gamma}\right)^{T}\left(B_{s}^{j}\right)^{T}\right] d s } \\
& +\left[\sum_{j=1}^{d} B_{s}^{j} y_{s}^{t, \eta}\left(y_{s}^{t, \gamma}\right)^{T}+y_{s}^{t, \eta}\left(y_{s}^{t, \gamma}\right)^{T}\left(B_{s}^{j}\right)^{T}\right] d W_{s}^{j} .
\end{aligned}
$$

Suppose for a moment that we are able to find a solution to equation (45) in $L_{\mathcal{F}}^{2,-r}\left(\mathbb{R}_{+} ; \mathcal{S}^{n}\right) \times$ $\left(L_{\mathcal{F}}^{2,-r}\left(\mathbb{R}_{+} ; \mathcal{S}^{n}\right)\right)^{d}$ for some $r>0$. Noting that $\left\langle P_{s} y_{s}^{t, \eta}, y_{s}^{t, \gamma}\right\rangle=\operatorname{Tr}\left\{P_{s} y_{s}^{t, \eta}\left(y_{s}^{t, \gamma}\right)^{T}\right\}$ and using equation (53), it follows by the Itô formula that for all $[t, T]$ we have $\mathbb{P}$-almost surely

$$
\begin{aligned}
e^{-r t}\left\langle P_{t} \eta, \gamma\right\rangle & =\mathbb{E}^{\mathcal{F}_{t}}\left[e^{-r t}\left\langle P_{t} y_{t}^{t, \eta}, y_{t}^{t, \gamma}\right\rangle\right] \\
& =\mathbb{E}^{\mathcal{F}_{t}}\left[e^{-r T}\left\langle P_{T} y_{T}^{t, \eta}, y_{T}^{t, \gamma}\right\rangle\right]+\mathbb{E}^{\mathcal{F}_{t}} \int_{t}^{T} e^{-r s}\left\langle D_{x}^{2} H(s) y_{s}^{t, \eta}, y_{s}^{t, \gamma}\right\rangle d s,
\end{aligned}
$$


where we have used the notation $D_{x}^{2} H(t):=D_{x}^{2} H\left(\bar{X}_{t}, \bar{u}_{t}, p_{t}, q_{t}\right)$ for the forcing term in the equation for $P$. Since the processes $P(\cdot)$ and $y{ }^{t, \eta}\left(y .{ }^{t, \gamma}\right)^{T}$ are assumed to be in some appropriate exponentiallyweighted spaces, there has to be a sequence of times $\left(T_{n}\right)_{n \geq 1}$ with $T_{n} \nearrow+\infty$ as $n \rightarrow+\infty$ such that $\mathbb{P}$-almost surely

$$
\lim _{n \rightarrow+\infty} \mathbb{E}^{\mathcal{F}_{t}}\left[e^{-r T_{n}}\left\langle P_{T_{n}} y_{T_{n}}^{t, \eta}, y_{T_{n}}^{t, \gamma}\right\rangle\right]=0 .
$$

Passing to the limit along the above sequence $\left(T_{n}\right)_{n \geq 1}$ produces the following formal relation

$$
\left\langle P_{t} \eta, \gamma\right\rangle=\mathbb{E}^{\mathcal{F}_{t}} \int_{t}^{\infty} e^{-r(s-t)}\left\langle D_{x}^{2} H(s) y_{s}^{t, \eta}, y_{s}^{t, \gamma}\right\rangle d s
$$

which can be used to define the process $P_{t}$. Our aim is to show that the right hand side of (56) actually defines a continuous bilinear form that can be used to prove (51) without any reference to the second adjoint BSDE.

Existence of $P$ : The following estimates on $\left(y_{s}^{t, \eta}\right)_{s \geq t}$ are crucial to prove continuity of the bilinear form.

Proposition 4. Let $\eta \in \mathbb{R}^{n}$ and assume that Hypotheses (H1)-(H4) hold. Then there is a unique solution $\left(y_{s}^{t, \eta}\right)_{s \geq t} \in L_{\mathcal{F}}^{2,-r}\left(\mathbb{R}_{+} ; \mathbb{R}^{n}\right)$ to the equation (52) for some $r$. Moreover, there exists a constant $C>0$ such that for $t \geq 0$ and $s \geq t$

$$
\sup _{s \geq t} \mathbb{E}^{\mathcal{F}_{t}}\left[e^{-r t}\left|y_{s}^{t, \eta}\right|^{4}\right] \leq C|\eta|^{4}, \quad \mathbb{P}-\text { a.s. }
$$

and for all $h>0,0 \leq t \leq t+h$ and $s \geq t+h$

$$
e^{-r s} \mathbb{E}\left|y_{s}^{t+h, \eta}-y_{s}^{t, \eta}\right|^{4} \leq C h .
$$

Proof. Let us choose $r>2 c_{1 / 2}$. The existence follows immediately by Theorem 2 and the proof of (57) it is a easy consequence of Lemma 1 with the additional requirement $r>2 \max \left\{c_{1 / 2}, c_{3}\right\}$. To prove the continuity property (58) let us denote $z_{s}=y_{s}^{t+h, \eta}-y_{s}^{t, \eta}$ then, for $s \geq t+h$, we have by the Itô formula

$$
\begin{aligned}
e^{-r s} \mathbb{E}\left|z_{s}\right|^{4}= & e^{-r(t+h)} \mathbb{E}\left|\eta-y_{t+h}^{t, \eta}\right|^{4}-r \mathbb{E} \int_{t+h}^{s} e^{-r \tau}\left|z_{\tau}\right|^{4} d \tau \\
& +\mathbb{E} \int_{t+h}^{s} e^{-r \tau}\left|z_{\tau}\right|^{2}\left\langle D_{x} b\left(\bar{X}_{\tau}, \bar{u}_{\tau}\right) z_{\tau}, z_{\tau}\right\rangle d \tau \\
& +\sum_{j=1}^{d} \mathbb{E} \int_{t+h}^{s} e^{-r \tau}\left\langle D_{x} \sigma^{j}\left(\bar{X}_{\tau}, \bar{u}_{\tau}\right) z_{\tau}, z_{\tau}\right\rangle^{2} d \tau \\
& +\sum_{j=1}^{d} \mathbb{E} \int_{t+h}^{s} e^{-r \tau}\left|z_{\tau}\right|^{2}\left\langle D_{x} \sigma^{j}\left(\bar{X}_{\tau}, \bar{u}_{\tau}\right) z_{\tau}, z_{\tau}\right\rangle d \tau
\end{aligned}
$$

Using the same estimate of Lemma 1 we end up with

$$
e^{-r s} \mathbb{E}\left|y_{s}^{t+h, \eta}-y_{s}^{t, \eta}\right|^{4} \leq K e^{-r(t+h)} \mathbb{E}\left|\eta-y_{t+h}^{t, \eta}\right|^{4},
$$

which we can control in the following form

$$
\begin{aligned}
\mathbb{E}\left|\eta-y_{t+h}^{t, \eta}\right|^{4} & =\mathbb{E}\left|\int_{t}^{t+h} D_{x} b\left(\bar{X}_{\tau}, \bar{u}_{\tau}\right) y_{\tau}^{t, \eta} d \tau+\sum_{j=1}^{d} \int_{t}^{t+h} D_{x} \sigma^{j}\left(\bar{X}_{\tau}, \bar{u}_{\tau}\right) y_{\tau}^{t, \eta} d W_{\tau}^{j}\right|^{4} \\
& \leq C \mathbb{E} \int_{t}^{t+h}\left|D_{x} b\left(\bar{X}_{\tau}, \bar{u}_{\tau}\right) y_{\tau}^{t, \eta}\right|^{4} d \tau+\sum_{j=1}^{d} \int_{t}^{t+h}\left|D_{x} \sigma^{j}\left(\bar{X}_{\tau}, \bar{u}_{\tau}\right) y_{\tau}^{t, \eta}\right|^{4} d \tau .
\end{aligned}
$$


Now, using Hölder inequality and again Lemma \ for the first term we obtain

$$
\begin{aligned}
\mathbb{E} \int_{t}^{t+h} & \left|D_{x} b\left(\bar{X}_{\tau}, \bar{u}_{\tau}\right) y_{\tau}^{t, \eta}\right|^{4} d \tau \\
& \leq\left(\mathbb{E} \int_{t}^{t+h} e^{r \tau} e^{-r \tau}|| D_{x} b\left(\bar{X}_{\tau}, \bar{u}_{\tau}\right)||^{8} d \tau\right)^{\frac{1}{2}}\left(\mathbb{E} \int_{t}^{t+h} e^{r \tau} e^{-r \tau}\left|y_{\tau}^{t, \eta}\right|^{8} d \tau\right)^{\frac{1}{2}} \\
& \leq h\left(\sup _{\tau \in[t, t+h]}\left(e^{-r \tau} \mathbb{E}\left|y_{\tau}^{t, \eta}\right|^{8}\right)\right)^{\frac{1}{2}}\left(\sup _{\tau \in[t, t+h]}\left(\left.e^{-r \tau} \mathbb{E}|| D_{x} b\left(\bar{X}_{\tau}, \bar{u}_{\tau}\right)\right|^{8}\right)\right)^{\frac{1}{2}} \\
& \leq C h .
\end{aligned}
$$

The $D_{x} \sigma$ term can be treated in the same way and the conclusion follows.

Proposition 5. Let Hypotheses (H1)-(H5) hold and $\gamma, \eta \in \mathbb{R}^{n}$. Then there exist $r>0$ and a progressive process $\left(P_{t}\right)_{t \geq 0}$ with values in $\mathcal{S}^{n}$ such that for all $t \geq 0$ it holds

$$
\left\langle P_{t} \eta, \gamma\right\rangle=\mathbb{E}^{\mathcal{F}_{t}} \int_{t}^{\infty} e^{-r(s-t)}\left\langle D_{x}^{2} H(s) y_{s}^{t, \eta}, y_{s}^{t, \gamma}\right\rangle d s, \quad \mathbb{P}-a . s .
$$

Moreover, $\sup _{t \geq 0} \mathbb{E}\left\|P_{t}\right\|^{2}<\infty$ and for $\varepsilon \searrow 0$ we have that

$$
\mathbb{E}\left|\left\langle\left(P_{t+\varepsilon}-P_{t}\right) \gamma, \eta\right\rangle\right| \rightarrow 0
$$

Proof. For $\gamma$ and $\eta \in \mathbb{R}^{n}$ fixed, let us define $\left\langle P_{t} \gamma, \eta\right\rangle$ by the formula given in the statement. To do so we have chosen an arbitrary version of the conditional expectation. To construct the process $P_{t}$ we have to prove that the map $(\gamma, \eta) \mapsto\left\langle P_{t} \gamma, \eta\right\rangle$ is a continuous bilinear form. Note that

$$
\begin{aligned}
& \left|\mathbb{E}^{\mathcal{F}_{t}} \int_{t}^{\infty} e^{-r(s-t)}\left\langle D_{x}^{2} H(s) y_{s}^{t, \eta}, y_{s}^{t, \gamma}\right\rangle d s\right| \\
& \leq \mathbb{E}^{\mathcal{F}_{t}} \int_{t}^{\infty} e^{-r(s-t)}\left|D_{x}^{2} H(s)\right|\left|y_{s}^{t, \eta}\right|\left|y_{s}^{t, \gamma}\right| d s \\
& \leq C \int_{t}^{\infty}\left(e^{-r(s-t)} \mathbb{E}^{\mathcal{F}_{t}}\left|D_{x}^{2} H(s)\right|^{p}\right)^{1 / p}\left(e^{-r(s-t)} \mathbb{E}^{\mathcal{F}_{t}}\left|y_{s}^{t, \eta}\right|^{2 q}\right)^{\frac{1}{2 q}}\left(e^{-r(s-t)} \mathbb{E}^{\mathcal{F}_{t}}\left|y_{s}^{t, \gamma}\right|^{2 q}\right)^{\frac{1}{2 q}} d s \\
& \leq C|\eta \| \gamma|\left(\int_{t}^{\infty} e^{-r(s-t)} \mathbb{E}^{\mathcal{F}_{t}}\left|D_{x}^{2} H(s)\right|^{p} d s\right)^{1 / p},
\end{aligned}
$$

where we used conditional Hölder inequality with $p \in(1,2), q=\frac{p}{p-1}>2$ and estimate (57). $r>0$ can be chosen such that $\int_{t}^{\infty} e^{-r s} \mathbb{E}^{\mathcal{F}_{t}}\left|D_{x}^{2} H(s)\right|^{p} d s<\infty$. This can be seen from the definition of the Hamiltonian, the estimates on first adjoint processes and the polynomial growth of the coefficients. Further, there exists a set $N$ such that $\mathbb{P}(N)=0$ and for $\omega \notin N$,

$$
\left|\left\langle P_{t}(\omega) \eta, \gamma\right\rangle\right| \leq C|\eta||\gamma| .
$$

If we set $P_{t}(\omega)=0$ for $\omega \in N$, by now we have constructed an adapted process $P_{t}$ which satisfies equation (61). The symmetry of the process $P$ is obtained easily by symmetry of $D_{x}^{2} H(s)$.

To construct a progressive modification of $P_{t}$, it is sufficient to prove that the map $(\omega, t) \mapsto P_{t}(\omega)$ is $\mathcal{F} \otimes \mathcal{B}\left(\mathbb{R}_{+}\right) \backslash \mathcal{B}\left(\mathbb{R}^{n \times n}\right)$-measurable (i.e. it is a jointly measurable process). Here, $\mathcal{B}\left(\mathbb{R}^{n \times n}\right)$ stands for a Borel $\sigma$-field induced by the norm $\|\cdot\|_{2}$ on $\mathbb{R}^{n \times n}$. If we prove that $P$ is an $\left(\mathcal{F}_{t}\right)$-adapted and jointly measurable process then there is an $\left(\mathcal{F}_{t}\right)$-progressive version of $P$. For a recent and elegant proof of this fact see [14]. Concerning joint measurability of $P$, its proof is given in [7]. In that paper, in fact, even the existence of a progressive version in infinite dimensional setting is provided without any reference to the classical result. 
To show that (62) holds, let us write

$$
\begin{aligned}
\left\langle\left(P_{t+\varepsilon}-P_{t}\right) \eta, \gamma\right\rangle= & \left(\mathbb{E}^{\mathcal{F}_{t+\varepsilon}}-\mathbb{E}^{\mathcal{F}_{t}}\right) \int_{t}^{\infty} e^{-r(s-t)}\left\langle D_{x}^{2} H(s) y_{s}^{t, \eta}, y_{s}^{t, \gamma}\right\rangle d s \\
& -\mathbb{E}^{\mathcal{F}_{t+\varepsilon}} \int_{t}^{t+\varepsilon} e^{-r(s-t)}\left\langle D_{x}^{2} H(s) y_{s}^{t, \eta}, y_{s}^{t, \gamma}\right\rangle d s \\
& +\mathbb{E}^{\mathcal{F}_{t+\varepsilon}} \int_{t+\varepsilon}^{\infty} e^{-r(s-t-\varepsilon)}\left(\left\langle D_{x}^{2} H(s) y_{s}^{t+\varepsilon, \eta}, y_{s}^{t+\varepsilon, \gamma}\right\rangle-\left\langle D_{x}^{2} H(s) y_{s}^{t, \eta}, y_{s}^{t, \gamma}\right\rangle\right) d s \\
& +\mathbb{E}^{\mathcal{F}_{t+\varepsilon}} \int_{t+\varepsilon}^{\infty}\left(e^{-r(s-t-\varepsilon)}-e^{-r(s-t)}\right)\left\langle D_{x}^{2} H(s) y_{s}^{t, \eta}, y_{s}^{t, \gamma}\right\rangle d s .
\end{aligned}
$$

The first summand on the right hand side goes to zero in $L^{1}(\Omega)$ as $\varepsilon \searrow 0$ thanks to the Lévy downward martingale convergence theorem (note that we have UC filtration $\left(\mathcal{F}_{t}\right)_{t>0}$ ), the second one and the last one tend to zero in $L^{1}(\Omega)$ by dominated convergence theorem. Regarding the third term the result easily follows by using (58). Indeed we can rewrite it as follows

$$
\begin{aligned}
& \mathbb{E}^{\mathcal{F}_{t+\varepsilon}} \int_{t+\varepsilon}^{\infty} e^{-r(s-t-\varepsilon)}\left\langle D_{x}^{2} H(s)\left(y_{s}^{t+\varepsilon, \eta}-y_{s}^{t, \eta}\right), y_{s}^{t+\varepsilon, \gamma}\right\rangle d s \\
& +\mathbb{E}^{\mathcal{F}_{t+\varepsilon}} \int_{t+\varepsilon}^{\infty} e^{-r(s-t-\varepsilon)}\left\langle D_{x}^{2} H(s) y_{s}^{t, \eta}, y_{s}^{t+\varepsilon, \gamma}-y_{s}^{t, \gamma}\right\rangle d s=I_{1}+I_{2} .
\end{aligned}
$$

Using Hölder inequality with $p \in(1,2), q=\frac{p}{p-1}>2$, the first addendum $I_{1}$ can be estimate by

$$
\begin{gathered}
I_{1} \leq e^{t+\varepsilon} \int_{t+\varepsilon}^{\infty}\left(e^{-r s} \mathbb{E}^{\mathcal{F}_{t+\varepsilon}}\left|D_{x}^{2} H(s)\right|^{p}\right)^{1 / p}\left(e^{-r s} \mathbb{E}^{\mathcal{F}_{t+\varepsilon}}\left|y_{s}^{t+\varepsilon, \eta}-y_{s}^{t, \eta}\right|^{2 q}\right)^{\frac{1}{2 q}} \\
\cdot\left(e^{-r s} \mathbb{E}^{\mathcal{F}_{t+\varepsilon}}\left|y_{s}^{t+\varepsilon, \gamma}\right|^{2 q}\right)^{\frac{1}{2 q}} d s
\end{gathered}
$$

Repeating the same estimate for the second addendum $I_{2}$, using Lemma 1 and (58) we get the required result.

Remark 5. If $F, G$ are random variables in $L^{2}(\Omega)$ measurable with respect to $\mathcal{F}_{t}$ then it is true that

$$
\left\langle P_{t} F, G\right\rangle=\mathbb{E}^{\mathcal{F}_{t}} \int_{t}^{\infty} e^{-r(s-t)}\left\langle D_{x}^{2} H(s) y_{s}^{t, F}, y_{s}^{t, G}\right\rangle d s, \quad \mathbb{P}-\text { a.s. }
$$

The proof follows by applying similar procedure as in Peng and Shi, [18], Theorem 13.

Proposition 6. Let $\left(y_{t}^{\varepsilon}\right)_{t \geq 0}$ be a solution to the first variation equation (27). Then there exists $r>0$ such that the following relations hold true.

$$
\begin{aligned}
& \text { i) } e^{-r\left(t_{0}+\varepsilon\right)} \mathbb{E}\left\langle\left(P_{t_{0}+\varepsilon}-P_{t_{0}}\right) y_{t_{0}+\varepsilon}^{\varepsilon}, y_{t_{0}+\varepsilon}^{\varepsilon}\right\rangle=o(\varepsilon), \\
& \text { ii) } \quad e^{-r\left(t_{0}+\varepsilon\right)} \mathbb{E}\left\langle P_{t_{0}} y_{t_{0}+\varepsilon}^{\varepsilon}, y_{t_{0}+\varepsilon}^{\varepsilon}\right\rangle \\
& \quad=\sum_{j=1}^{d} \mathbb{E} \int_{0}^{\infty} e^{-r s}\left\langle P_{s} \delta \sigma^{j}\left(\bar{X}_{s}, \bar{u}_{s}\right), \delta \sigma^{j}\left(\bar{X}_{s}, \bar{u}_{s}\right)\right\rangle d s+o(\varepsilon) .
\end{aligned}
$$

Proof. (i) From Proposition 1-(ii) we know that there exists $r$ such that

$$
\left(e^{-r\left(t_{0}+\varepsilon\right)} \mathbb{E}\left|\varepsilon^{1 / 2} y_{t_{0}+\varepsilon}^{\varepsilon}\right|^{2 k}\right)^{1 / 2 k} \leq C, \quad k \geq 1,
$$

and by the Markov inequality, for every $\delta>0$ we have that

$$
\mathbb{P}\left(\left|\varepsilon^{1 / 2} y_{t_{0}+\varepsilon}^{\varepsilon}\right|>C \delta^{-1 / 4}\right) \leq e^{r\left(t_{0}+\varepsilon\right)} \delta .
$$

If we denote $\Omega_{\delta, \varepsilon}$ the event $\left\{\varepsilon^{-1 / 2} y_{t_{0}+\varepsilon}^{\varepsilon} \in B_{C \delta^{-1 / 4}}\right\}$, where $B_{C \delta^{-1 / 4}}$ is the centred ball with radius $\delta^{-1 / 4}$, then it holds

$$
\mathbb{P}\left(\Omega_{\delta, \varepsilon}^{c}\right) \leq e^{r\left(t_{0}+\varepsilon\right)} \delta
$$


Now we rewrite (i) in the following form

$$
\begin{aligned}
e^{-r\left(t_{0}+\varepsilon\right)} \mathbb{E}\left\langle\left(P_{t_{0}+\varepsilon}-P_{t_{0}}\right) \varepsilon^{-1 / 2} y_{t_{0}+\varepsilon}^{\varepsilon}, \varepsilon^{-1 / 2} y_{t_{0}+\varepsilon}^{\varepsilon}\right\rangle \\
=e^{-r\left(t_{0}+\varepsilon\right)} \mathbb{E}\left(\left\langle\left(P_{t_{0}+\varepsilon}-P_{t_{0}}\right) \varepsilon^{-1 / 2} y_{t_{0}+\varepsilon}^{\varepsilon}, \varepsilon^{-1 / 2} y_{t_{0}+\varepsilon}^{\varepsilon}\right\rangle 1_{\Omega_{\delta, \varepsilon}^{c}}\right) \\
\quad+e^{-r\left(t_{0}+\varepsilon\right)} \mathbb{E}\left(\left\langle\left(P_{t_{0}+\varepsilon}-P_{t_{0}}\right) \varepsilon^{-1 / 2} y_{t_{0}+\varepsilon}^{\varepsilon}, \varepsilon^{-1 / 2} y_{t_{0}+\varepsilon}^{\varepsilon}\right\rangle 1_{\Omega_{\delta, \varepsilon}}\right) \\
=: A_{1}^{\varepsilon}+A_{2}^{\varepsilon} .
\end{aligned}
$$

The first term can be easily treated by the Hölder inequality, Proposition 5 and estimates (66), (67), respectively. Hence, the estimate reads

$$
\begin{aligned}
\left|A_{1}^{\varepsilon}\right| \leq & \left(e^{-r\left(t_{0}+\varepsilon\right)} \mathbb{E}\left\|P_{t_{0}+\varepsilon}-P_{t_{0}}\right\|^{2}\right)^{1 / 2} \\
& \cdot\left(e^{-r\left(t_{0}+\varepsilon\right)} \mathbb{E}\left|\varepsilon^{-1 / 2} y_{t_{0}+\varepsilon}^{\varepsilon}\right|^{8}\right)^{1 / 4}\left(e^{-r\left(t_{0}+\varepsilon\right)} \mathbb{P}\left(\Omega_{\delta, \varepsilon}^{c}\right)\right)^{1 / 4} \\
\leq & C \delta^{1 / 4}
\end{aligned}
$$

Regarding the second term, we have that

$$
\left|A_{2}^{\varepsilon}\right| \leq e^{-r\left(t_{0}+\varepsilon\right)} \mathbb{E}\left[\sup _{x \in B_{C \delta^{-1 / 4}}}\left|\left\langle\left(P_{t_{0}+\varepsilon}-P_{t_{0}}\right) x, x\right\rangle 1_{\Omega_{\delta, \varepsilon}}\right|\right] .
$$

Since $B_{C \delta^{-1 / 4}}$ is compact, there exist $N_{\delta}$ open balls $B_{x_{i}, \delta}$ which cover it. Moreover, for all $x \in$ $B_{C \delta^{-1 / 4}}$ we can choose $i$ such that $\left|x-x_{i}\right| \leq \delta$. Then

$$
\begin{aligned}
\left\langle\left(P_{t_{0}+\varepsilon}-P_{t_{0}}\right) x, x\right\rangle= & \left\langle\left(P_{t_{0}+\varepsilon}-P_{t_{0}}\right) x_{i}, x_{i}\right\rangle-\left\langle\left(P_{t_{0}+\varepsilon}-P_{t_{0}}\right)\left(x-x_{i}\right),\left(x-x_{i}\right)\right\rangle \\
& +2\left\langle\left(P_{t_{0}+\varepsilon}-P_{t_{0}}\right) x,\left(x-x_{i}\right)\right\rangle \\
= & \left\langle\left(P_{t_{0}+\varepsilon}-P_{t_{0}}\right) x_{i}, x_{i}\right\rangle+\left\|P_{t_{0}+\varepsilon}-P_{t_{0}}\right\|_{\infty} \delta^{2} \\
& +2\left\|P_{t_{0}+\varepsilon}-P_{t_{0}}\right\|_{\infty}|x| \delta
\end{aligned}
$$

where, for a generic matrix $T \in \mathbb{R}^{n \times n}$, we have used $\|T\|_{\infty}:=\sup \left\{|\langle T x, y\rangle|: x, y \in \mathbb{R}^{n},|x| \leq\right.$ $1,|y| \leq 1\}$. Taking supremum and expectation we obtain

$$
\left|A_{2}^{\varepsilon}\right| \leq \sum_{i=1}^{N_{\delta}} \mathbb{E}\left|\left\langle\left(P_{t_{0}+\varepsilon}-P_{t_{0}}\right) x_{i}, x_{i}\right\rangle\right|+C\left(\delta^{2}+\delta^{3 / 4}\right) .
$$

If we let $\varepsilon \rightarrow 0$ and use (62) it follows that

$$
\limsup _{\varepsilon \rightarrow 0}\left|A_{2}^{\varepsilon}\right| \leq C\left(\delta^{2}+\delta^{3 / 4}\right)
$$

hence $\left|A_{1}^{\varepsilon}\right|+\left|A_{2}^{\varepsilon}\right| \rightarrow 0$, when $\delta \rightarrow 0$ and the proof of (i) is finished.

(ii) Let us rewrite $e^{-r\left(t_{0}+\varepsilon\right)} \mathbb{E}\left\langle P_{t_{0}} y_{t_{0}+\varepsilon}^{\varepsilon}, y_{t_{0}+\varepsilon}^{\varepsilon}\right\rangle$ in the following form

$$
e^{-r\left(t_{0}+\varepsilon\right)} \mathbb{E}\left\langle P_{t_{0}} y_{t_{0}+\varepsilon}^{\varepsilon}, y_{t_{0}+\varepsilon}^{\varepsilon}\right\rangle=\mathbb{E}\left[\operatorname{Tr}\left\{P_{t_{0}}\left(e^{-\frac{r}{2}\left(t_{0}+\varepsilon\right)} y_{t_{0}+\varepsilon}^{\varepsilon}\right)\left(e^{-\frac{r}{2}\left(t_{0}+\varepsilon\right)} y_{t_{0}+\varepsilon}^{\varepsilon}\right)^{T}\right\}\right] .
$$

Thanks to the Itô formula and equation (47), we obtain

$$
\begin{aligned}
d\left(e^{-r s} Y_{s}^{\varepsilon}\right)= & e^{-r s}\left[-r Y_{s}^{\varepsilon}+D_{x} b\left(\bar{X}_{s}, \bar{u}_{s}\right) Y_{s}^{\varepsilon}+Y_{s}^{\varepsilon} D_{x} b\left(\bar{X}_{s}, \bar{u}_{s}\right)^{T}\right. \\
& \left.+\sum_{j=1}^{d} D_{x} \sigma^{j}\left(\bar{X}_{s}, \bar{u}_{s}\right) Y_{s}^{\varepsilon} D_{x} \sigma^{j}\left(\bar{X}_{s}, \bar{u}_{s}\right)^{T}+\Gamma(s)\right] d s \\
& +\sum_{j=1}^{d} e^{-r s}\left[D_{x} \sigma^{j}\left(\bar{X}_{s}, \bar{u}_{s}\right) Y_{s}^{\varepsilon}+Y_{s}^{\varepsilon} D_{x} \sigma^{j}\left(\bar{X}_{s}, \bar{u}_{s}\right)^{T}+\Lambda^{j}(s)\right] d W_{s}^{j},
\end{aligned}
$$

where we have used the notation $Y_{s}^{\varepsilon}=y_{s}^{\varepsilon}\left(y_{s}^{\varepsilon}\right)^{T}$ and $\Gamma(s), \Lambda^{j}(s)$ are as in (48), (49). Now, by taking conditional expectation with respect to $\mathcal{F}_{t_{0}}$ and rewriting the equation in integral form from $t_{0}$ to 
$s$ (recall Remark 2) it follows that

$$
\begin{aligned}
\mathbb{E}^{\mathcal{F}_{t_{0}}}\left(e^{-r s} Y_{s}^{\varepsilon}\right)= & \mathbb{E}^{\mathcal{F}_{t_{0}}} \int_{t_{0}}^{s} e^{-r \tau}\left[-r Y_{\tau}^{\varepsilon}+D_{x} b\left(\bar{X}_{\tau}, \bar{u}_{\tau}\right) Y_{\tau}^{\varepsilon}+Y_{\tau}^{\varepsilon} D_{x} b\left(\bar{X}_{\tau}, \bar{u}_{\tau}\right)^{T}\right] d \tau \\
& +\sum_{j=1}^{d} \mathbb{E}^{\mathcal{F}_{t_{0}}} \int_{t_{0}}^{s}\left[D_{x} \sigma^{j}\left(\bar{X}_{\tau}, \bar{u}_{\tau}\right) Y_{\tau}^{\varepsilon} D_{x} \sigma^{j}\left(\bar{X}_{\tau}, \bar{u}_{\tau}\right)^{T}+\Gamma(\tau)\right] d \tau
\end{aligned}
$$

Hence, taking into account the definition of $\Gamma$ in (48), multiplying by $P_{t_{0}}$, setting $s=t_{0}+\varepsilon$ and taking expectation, we arrive at

$$
\begin{aligned}
& e^{-r\left(t_{0}+\varepsilon\right)} \mathbb{E}\left\langle P_{t_{0}} y_{t_{0}+\varepsilon}^{\varepsilon}, y_{t_{0}+\varepsilon}^{\varepsilon}\right\rangle \\
& =\int_{t_{0}}^{t_{0}+\varepsilon} e^{-r \tau} \mathbb{E}\left[\operatorname{Tr}\left\{P_{t_{0}}\left(-r Y_{\tau}^{\varepsilon}+D_{x} b\left(\bar{X}_{\tau}, \bar{u}_{\tau}\right) Y_{\tau}^{\varepsilon}+Y_{\tau}^{\varepsilon} D_{x} b\left(\bar{X}_{\tau}, \bar{u}_{\tau}\right)^{T}\right)\right\}\right] d \tau \\
& \quad+\sum_{j=1}^{d} \int_{t_{0}}^{t_{0}+\varepsilon} e^{-r \tau} \mathbb{E}\left[\operatorname{Tr}\left\{P_{t_{0}}\left(D_{x} \sigma^{j}\left(\bar{X}_{\tau}, \bar{u}_{\tau}\right) Y_{\tau}^{\varepsilon} D_{x} \sigma^{j}\left(\bar{X}_{\tau}, \bar{u}_{\tau}\right)^{T}\right)\right\}\right] d \tau \\
& \quad+\sum_{j=1}^{d} \int_{t_{0}}^{t_{0}+\varepsilon} e^{-r \tau} \mathbb{E}\left[\operatorname{Tr}\left\{P_{t_{0}}\left(\delta \sigma^{j}\left(\bar{X}_{\tau}, \bar{u}_{\tau}\right)\left(\delta \sigma^{j}\left(\bar{X}_{\tau}, \bar{u}_{\tau}\right)\right)^{T}\right)\right\}\right] d \tau \\
& \quad+\sum_{j=1}^{d} \int_{t_{0}}^{t_{0}+\varepsilon} e^{-r \tau} \mathbb{E}\left[\operatorname{Tr}\left\{P_{t_{0}}\left(D_{x} \sigma^{j}\left(\bar{X}_{\tau}, \bar{u}_{\tau}\right) y_{\tau}^{\varepsilon}\left(\delta \sigma^{j}\left(\bar{X}_{\tau}, \bar{u}_{\tau}\right)\right)^{T}\right)\right\}\right] d \tau \\
& \quad+\sum_{j=1}^{d} \int_{t_{0}}^{t_{0}+\varepsilon} e^{-r \tau} \mathbb{E}\left[\operatorname{Tr}\left\{P_{t_{0}}\left(\delta \sigma^{j}\left(\bar{X}_{\tau}, \bar{u}_{\tau}\right)\left(y_{\tau}^{\varepsilon}\right)^{T} D_{x} \sigma^{j}\left(\bar{X}_{\tau}, \bar{u}_{\tau}\right)^{T}\right)\right\}\right] d \tau .
\end{aligned}
$$

We will show that using the estimate for $y_{s}^{\varepsilon}$ in the form of (66), the only term which is not of order $o(\varepsilon)$ is the third one. Therefore, the final equality will read

$$
\begin{aligned}
& e^{-r\left(t_{0}+\varepsilon\right)} \mathbb{E}\left\langle P_{t_{0}} y_{t_{0}+\varepsilon}^{\varepsilon}, y_{t_{0}+\varepsilon}^{\varepsilon}\right\rangle \\
& =\sum_{j=1}^{d} \mathbb{E} \int_{t_{0}}^{t_{0}+\varepsilon} e^{-r \tau}\left\langle P_{t_{0}} \delta \sigma^{j}\left(\bar{X}_{\tau}, \bar{u}_{\tau}\right), \delta \sigma^{j}\left(\bar{X}_{\tau}, \bar{u}_{\tau}\right)\right\rangle d \tau+o(\varepsilon) .
\end{aligned}
$$

For sake of completeness, let us estimate the second term as

$$
\begin{aligned}
& \sum_{j=1}^{d} \int_{t_{0}}^{t_{0}+\varepsilon} e^{-r \tau} \mathbb{E}\left[\operatorname{Tr}\left\{P_{t_{0}}\left(D_{x} \sigma^{j}\left(\bar{X}_{\tau}, \bar{u}_{\tau}\right) Y_{\tau}^{\varepsilon} D_{x} \sigma^{j}\left(\bar{X}_{\tau}, \bar{u}_{\tau}\right)^{T}\right)\right\}\right] d \tau \\
& =\sum_{j=1}^{d} \int_{t_{0}}^{t_{0}+\varepsilon} e^{-r \tau} \mathbb{E}\left\langle P_{t_{0}} D_{x} \sigma^{j}\left(\bar{X}_{\tau}, \bar{u}_{\tau}\right) y_{\tau}^{\varepsilon}, D_{x} \sigma^{j}\left(\bar{X}_{\tau}, \bar{u}_{\tau}\right) y_{\tau}^{\varepsilon}\right\rangle d \tau \\
& \leq \sum_{j=1}^{d} \int_{t_{0}}^{t_{0}+\varepsilon} e^{-r \tau} \mathbb{E}\left[\left\|P_{t_{0}}\right\|_{2}\left|D_{x} \sigma^{j}\left(\bar{X}_{\tau}, \bar{u}_{\tau}\right)\right|^{2}\left|y_{\tau}^{\varepsilon}\right|^{2}\right] d \tau \\
& \leq \sum_{j=1}^{d} \int_{t_{0}}^{t_{0}+\varepsilon} \mathbb{E}\left(e^{-r \tau}\left\|P_{t_{0}}\right\|_{2}^{2}\right)^{1 / 2} \mathbb{E}\left(e^{-r \tau}\left|D_{x} \sigma^{j}\left(\bar{X}_{\tau}, \bar{u}_{\tau}\right)\right|^{4}\right)^{1 / 2} \mathbb{E}\left(e^{-r \tau}\left|y_{\tau}^{\varepsilon}\right|^{4}\right)^{1 / 2} d \tau,
\end{aligned}
$$

and the order of $o(\varepsilon)$ now follows by Proposition 5 , the polynomial growth of $D_{x} \sigma(\cdot)$ and estimate (66), respectively. The remaining terms can be treated in the similar way.

To finalize the proof of (72), it remains to be shown that

$$
\sum_{j=1}^{d} \mathbb{E} \int_{t_{0}}^{t_{0}+\varepsilon} e^{-r \tau}\left\langle\left(P_{\tau}-P_{t_{0}}\right) \delta \sigma^{j}\left(\bar{X}_{\tau}, \bar{u}_{\tau}\right), \delta \sigma^{j}\left(\bar{X}_{\tau}, \bar{u}_{\tau}\right)\right\rangle d \tau=o(\varepsilon) .
$$

But this is easily obtained by repeating the same arguments as in the proof of (i). The proof of the Proposition is now concluded. 


\section{NeCESSARY Stochastic MAXIMUM PRINCIPLE}

For our main result we need to recall the notion of the Hamiltonian of the system. Given the control problem (5)-(6), let us define $H: \mathbb{R}^{n} \times U \times \mathbb{R}^{n} \times \mathbb{R}^{n \times d} \rightarrow \mathbb{R}$ as

$$
H(x, u, p, q)=\langle p, b(x, u)\rangle+\operatorname{Tr}\left[q^{T} \sigma(x, u)\right]-f(x, u) .
$$

Theorem 5. Assume (H1)-(H5) hold and let $(\bar{X}, \bar{u})$ be an optimal pair. Then there exist $r>0, a$ pair $(p, q) \in L_{\mathcal{F}}^{2,-r}\left(\mathbb{R}_{+} ; \mathbb{R}^{n}\right) \times L_{\mathcal{F}}^{2,-r}\left(\mathbb{R}_{+} ; \mathbb{R}^{n \times d}\right)$ and a progressively measurable process $\left(P_{t}\right)_{t \geq 0}$ with values in $\mathcal{S}^{n}$ such that the following variational inequality holds, $\mathbb{P} \otimes d t$-a.e.

$H\left(\bar{X}_{t}, v, p_{t}, q_{t}\right)-H\left(\bar{X}_{t}, \bar{u}_{t}, p_{t}, q_{t}\right)+\frac{1}{2} \sum_{j=1}^{d}\left\langle P_{t}\left(\sigma^{j}\left(\bar{X}_{t}, v\right)-\sigma^{j}\left(\bar{X}_{t}, \bar{u}_{t}\right)\right), \sigma^{j}\left(\bar{X}_{t}, v\right)-\sigma^{j}\left(\bar{X}_{t}, \bar{u}_{t}\right)\right\rangle \leq 0$,

for every $v \in U$. The pair of processes $(p, q)$ is the unique solution to the first adjoint equation (35). The definition of the process $P_{t}$ is given in Proposition 5 and the process satisfies $\sup _{t \geq 0} \mathbb{E}\left\|P_{t}\right\|_{2}^{2}<$ $\infty$.

Remark 6. A sufficient condition for such $r$ is given in the Appendix.

Before proving the theorem, it is useful to rewrite the variation of cost functional in a suitable form, as the following proposition suggests.

Proposition 7. There exists $r>0$ such that the following expansion holds

$$
\begin{aligned}
J\left(u^{\varepsilon}(\cdot)\right)-J(\bar{u}(\cdot))= & \mathbb{E} \int_{0}^{\infty} e^{-r t}\left[-\sum_{j=1}^{d}\left\langle q_{t}^{j}, \delta \sigma^{j}\left(\bar{X}_{t}, \bar{u}_{t}\right)\right\rangle-\left\langle p_{t}, \delta b\left(\bar{X}_{t}, \bar{u}_{t}\right)\right\rangle+\delta f\left(\bar{X}_{t}, \bar{u}_{t}\right)\right] d t \\
& -\frac{1}{2} \mathbb{E} \int_{0}^{\infty} e^{-r t} \operatorname{Tr}\left[D_{x}^{2} H\left(\bar{X}_{t}, \bar{u}_{t}, p_{t}, q_{t}\right) y_{t}^{\varepsilon}\left(y_{t}^{\varepsilon}\right)^{T}\right] d t+o(\varepsilon),
\end{aligned}
$$

where $H\left(\bar{X}_{t}, \bar{u}_{t}, p_{t}, q_{t}\right)$ is the Hamiltonian of the system computed along the optimal trajectory.

Proof. See Appendix.

Now we are in position to end the proof of the SMP.

Proof of Theorem 可. The difficult step of the proof is to show that the following holds

$$
\mathbb{E} \int_{0}^{\infty} e^{-r s}\left\langle D_{x}^{2} H(s) y_{s}^{\varepsilon}, y_{s}^{\varepsilon}\right\rangle d s=\sum_{j=1}^{d} \mathbb{E} \int_{0}^{\infty} e^{-r s}\left\langle P_{s} \delta \sigma^{j}\left(\bar{X}_{s}, \bar{u}_{s}\right), \delta \sigma^{j}\left(\bar{X}_{s}, \bar{u}_{s}\right)\right\rangle d s+o(\varepsilon) .
$$

Indeed, if relation (76) holds true then by using Proposition 7 we get

$$
\begin{aligned}
0 \leq & J\left(u^{\varepsilon}(\cdot)\right)-J(\bar{u}(\cdot)) \\
= & \mathbb{E} \int_{0}^{\infty} e^{-r s}\left[-\sum_{j=1}^{d}\left\langle q_{s}^{j}, \delta \sigma^{j}\left(\bar{X}_{s}, \bar{u}_{s}\right)\right\rangle-\left\langle p_{s}, \delta b\left(\bar{X}_{s}, \bar{u}_{s}\right)\right\rangle+\delta f\left(\bar{X}_{s}, \bar{u}_{s}\right)\right] d t \\
& -\frac{1}{2} \sum_{j=1}^{d} \mathbb{E} \int_{0}^{\infty} e^{-r s}\left\langle P_{s} \delta \sigma^{j}\left(\bar{X}_{s}, \bar{u}_{s}\right), \delta \sigma^{j}\left(\bar{X}_{s}, \bar{u}_{s}\right)\right\rangle d s+o(\varepsilon),
\end{aligned}
$$

thanks to the optimality of $\bar{u}(\cdot)$. Now the final variational inequality follows by standard arguments, i.e. by using the definition of $\delta \sigma^{j}, \delta b, \delta f$, noting that $E_{\varepsilon}=\left[t_{0}, t_{0}+\varepsilon\right]$ and by sending $\varepsilon \searrow 0$.

Let us focus on the proof of (76). Recalling Remark 2, we can rewrite the left hand side of (76) in the following form

$$
\begin{aligned}
& \mathbb{E} \int_{0}^{\infty} e^{-r s}\left\langle D_{x}^{2} H(s) y_{s}^{\varepsilon}, y_{s}^{\varepsilon}\right\rangle d s \\
& \quad=\mathbb{E} \int_{t_{0}}^{t_{0}+\varepsilon} e^{-r s}\left\langle D_{x}^{2} H(s) y_{s}^{\varepsilon}, y_{s}^{\varepsilon}\right\rangle d s+\mathbb{E} \int_{t_{0}+\varepsilon}^{\infty} e^{-r s}\left\langle D_{x}^{2} H(s) y_{s}^{\varepsilon}, y_{s}^{\varepsilon}\right\rangle d s \\
& \quad=\mathbb{E} \int_{t_{0}+\varepsilon}^{\infty} e^{-r s}\left\langle D_{x}^{2} H(s) y_{s}^{t_{0}+\varepsilon, y_{t_{0}+\varepsilon}^{\varepsilon}}, y_{s}^{t_{0}+\varepsilon, y_{t_{0}+\varepsilon}^{\varepsilon}}\right\rangle d s+o(\varepsilon),
\end{aligned}
$$


where we have used Proposition 11 to estimate the first integral and the identity $y_{s}^{\varepsilon}=y_{s}^{t_{0}+\varepsilon, y_{t_{0}+\varepsilon}^{\varepsilon}}$, for $s \geq t_{0}+\varepsilon$. Taking into account Remark 5 , we finally deduce the following decomposition

$$
\begin{aligned}
\mathbb{E} \int_{0}^{\infty} e^{-r s}\left\langle D_{x}^{2} H(s) y_{s}^{\varepsilon}, y_{s}^{\varepsilon}\right\rangle d s= & e^{-r\left(t_{0}+\varepsilon\right)} \mathbb{E}\left\langle P_{t_{0}+\varepsilon} y_{t_{0}+\varepsilon}^{\varepsilon}, y_{t_{0}+\varepsilon}^{\varepsilon}\right\rangle+o(\varepsilon) \\
= & e^{-r\left(t_{0}+\varepsilon\right)} \mathbb{E}\left\langle\left(P_{t_{0}+\varepsilon}-P_{t_{0}}\right) y_{t_{0}+\varepsilon}^{\varepsilon}, y_{t_{0}+\varepsilon}^{\varepsilon}\right\rangle \\
& +e^{-r\left(t_{0}+\varepsilon\right)} \mathbb{E}\left\langle P_{t_{0}} y_{t_{0}+\varepsilon}^{\varepsilon}, y_{t_{0}+\varepsilon}^{\varepsilon}\right\rangle+o(\varepsilon) .
\end{aligned}
$$

Finally, by Proposition 6, the proof of the Theorem is now concluded.

\section{ACKNOWLEDGEMENTS}

The authors wish to thank to Marco Fuhrman and Gianmario Tessitore for encouragement and for many valuable discussions.

\section{APPENDIX}

\subsection{Proof of Proposition 1.}

Proof. In the following we are going to linearize the equations satisfied by $\xi^{\varepsilon}(\cdot), \eta^{\varepsilon}(\cdot)$ and $\zeta^{\varepsilon}(\cdot)$ in order to use the estimate obtained in Lemma 1.

(i) It is easy to see that the equation for $\xi^{\varepsilon}(\cdot)$ can be rewritten in the form

$$
d \xi_{t}^{\varepsilon}=\left[G_{b}(t) \xi_{t}^{\varepsilon}+\delta b_{t} \chi_{E_{\varepsilon}}(t)\right] d t+\sum_{j=1}^{d}\left[G_{\sigma}^{j}(t) \xi_{t}^{\varepsilon}+\delta \sigma_{t}^{j} \chi_{E_{\varepsilon}}(t)\right] d W_{t}^{j},
$$

where

$$
G_{b}(t):=\int_{0}^{1} D_{x} b\left(\bar{X}_{t}+\theta \xi_{t}^{\varepsilon}, u_{t}^{\varepsilon}\right) d \theta, \quad G_{\sigma}^{j}(t):=\int_{0}^{1} D_{x} \sigma^{j}\left(\bar{X}_{t}+\theta \xi_{t}^{\varepsilon}, u_{t}^{\varepsilon}\right) d \theta .
$$

Thanks to Hypothesis (H4) we can apply Lemma 1 and obtain (the constant $K>0$ varies from line to line)

$$
\begin{aligned}
\sup _{t \in \mathbb{R}_{+}} e^{-r k t} \mathbb{E}\left|\xi_{t}^{\varepsilon}\right|^{2 k} \leq & K\left[\int_{0}^{\infty} e^{-\frac{r}{2} t}\left(\mathbb{E}\left|\delta b_{t} \chi_{E_{\varepsilon}}(t)\right|^{2 k}\right)^{\frac{1}{2 k}} d t\right]^{2 k} \\
& +K \sum_{j=1}^{d}\left[\int_{0}^{\infty} e^{-r t}\left(\mathbb{E}\left|\delta \sigma_{t}^{j} \chi_{E_{\varepsilon}}(t)\right|^{2 k}\right)^{\frac{1}{k}} d t\right]^{k} \\
\leq & K\left[\int_{E_{\varepsilon}} e^{-\frac{r}{2} t}\left(\mathbb{E}\left|b\left(\bar{X}_{t}, u_{t}^{\varepsilon}\right)-b\left(\bar{X}_{t}, \bar{u}_{t}\right)\right|^{2 k}\right)^{\frac{1}{2 k}} d t\right]^{2 k} \\
& +K \sum_{j=1}^{d}\left[\int_{E_{\varepsilon}} e^{-r t}\left(\mathbb{E}\left|\sigma_{t}^{j}\left(\bar{X}_{t}, u_{t}^{\varepsilon}\right)-\sigma_{t}^{j}\left(\bar{X}_{t}, \bar{u}_{t}\right)\right|^{2 k}\right)^{\frac{1}{k}} d t\right]^{k} \\
\leq & K\left[\varepsilon^{2 k}+\varepsilon^{k}\right] \leq K \varepsilon^{k},
\end{aligned}
$$

thanks to the polynomial growth of the coefficients and the boundedness of the integration interval $E_{\varepsilon}$. Indeed, remember that it is easy to control all the moments of $\bar{X}$ up to a fixed time. In this case the discount factor $\rho_{1}$ can be chosen equal to the initial one $\rho_{1}=r$.

(ii) Using again the global monotonicity assumption and Lemma 1, the estimate for $y^{\varepsilon}$ follows in the same way.

(iii) For $z^{\varepsilon}$ we start by estimating its norm in the space $L_{\mathcal{F}}^{2 k,-r k \alpha}\left(\mathbb{R}_{+} ; \mathbb{R}^{n}\right)$, for a generic $\alpha \in \mathbb{R}$. 
Using the same technique as in Lemma 1, we obtain

$$
\begin{aligned}
\sup _{t \in \mathbb{R}_{+}} e^{-r k \alpha t} \mathbb{E}\left|z_{t}^{\varepsilon}\right|^{2 k} \leq & K\left[\int_{0}^{\infty} e^{-\frac{r \alpha}{2} t}\left(\mathbb{E}\left|\delta b_{t} \chi_{E_{\varepsilon}}(t)+\frac{1}{2} D_{x}^{2} b\left(\bar{X}_{t}, \bar{u}_{t}\right)\left(y_{t}^{\varepsilon}\right)^{2}\right|^{2 k}\right)^{\frac{1}{2 k}} d t\right]^{2 k} \\
& +K \sum_{j=1}^{d}\left[\int_{0}^{\infty} e^{-r \alpha t}\left(\mathbb{E}\left|\delta\left(D_{x} \sigma_{t}^{j}\right) \chi_{E_{\varepsilon}}(t) y_{t}^{\varepsilon}+\frac{1}{2} D_{x}^{2} \sigma^{j}\left(\bar{X}_{t}, \bar{u}_{t}\right)\left(y_{t}^{\varepsilon}\right)^{2}\right|^{2 k}\right)^{\frac{1}{k}} d t\right]^{k}
\end{aligned}
$$

The first term (with $\delta b_{t}$ ) can be treated as before, thanks to the boundedness of $E_{\varepsilon}$. Let us discuss the second one. It holds

$$
\begin{aligned}
& \int_{0}^{\infty} e^{-\frac{r \alpha}{2} t}\left(\mathbb{E}\left|D_{x}^{2} b\left(\bar{X}_{t}, \bar{u}_{t}\right)\left(y_{t}^{\varepsilon}\right)^{2}\right|^{2 k}\right)^{\frac{1}{2 k}} d t \\
& \leq \int_{0}^{\infty}\left(e^{-r \alpha k t} \mathbb{E}\left|D_{x}^{2} b\left(\bar{X}_{t}, \bar{u}_{t}\right)\right|^{4 k}\right)^{\frac{1}{4 k}}\left(e^{-r \alpha k t} \mathbb{E}\left|y_{t}^{\varepsilon}\right|^{8 k}\right)^{\frac{1}{4 k}} d t \\
& \leq\left(\sup _{t \geq 0} e^{-r \alpha k t} \mathbb{E}\left|y_{t}^{\varepsilon}\right|^{8 k}\right)^{\frac{1}{4 k}} \int_{0}^{\infty} e^{-\frac{r \alpha}{4} t}\left(\mathbb{E}\left|D_{x}^{2} b\left(\bar{X}_{t}, \bar{u}_{t}\right)\right|^{4 k}\right)^{\frac{1}{4 k}} d t \\
& \leq K\left(\sup _{t \geq 0} e^{-r \alpha k t} \mathbb{E}\left|y_{t}^{\varepsilon}\right|^{8 k}\right)^{\frac{1}{4 k}} \int_{0}^{\infty} e^{-\frac{r \alpha}{4} t}\left(\left.\left.\mathbb{E}|1+| \bar{X}_{t}\right|^{2 m+1}\right|^{4 k}\right)^{\frac{1}{4 k}} d t \\
& \leq K\left(\sup _{t \geq 0} e^{-r \alpha k t} \mathbb{E}\left|y_{t}^{\varepsilon}\right|^{8 k}\right)^{\frac{1}{4 k}}\left[\int_{0}^{\infty} e^{-\frac{r \alpha}{4} t} d t+\int_{0}^{\infty} e^{-\frac{r \alpha}{4} t}\left(\mathbb{E}\left|\bar{X}_{t}\right|^{4 k(2 m+1)}\right)^{\frac{1}{4 k}} d t\right] \\
& \leq K\left(\sup _{t \geq 0} e^{-r \alpha k t} \mathbb{E}\left|y_{t}^{\varepsilon}\right|^{8 k}\right)^{\frac{1}{4 k}}\left[\int_{0}^{\infty} e^{-\frac{r \alpha}{4} t} d t+\left(\int_{0}^{\infty} e^{-\frac{r \alpha}{4} t} \mathbb{E}\left|\bar{X}_{t}\right|^{4 k(2 m+1)} d t\right)^{\frac{1}{4 k}}\right]
\end{aligned}
$$

where we used the polynomial growth of $D_{x}^{2} b$ and Jensen inequality, assuming that $\int_{0}^{\infty} e^{-\frac{r \alpha}{4} t} d t<\infty$, hence $r \alpha>0$. Moreover, if we choose $\alpha \geq \max (4,8 k(2 m+1))=8 k(2 m+1)$, we have that

$$
\int_{0}^{\infty} e^{-\frac{r \alpha}{2} t}\left(\mathbb{E}\left|D_{x}^{2} b\left(\bar{X}_{t}, \bar{u}_{t}\right)\left(y_{t}^{\varepsilon}\right)^{2}\right|^{2 k}\right)^{\frac{1}{2 k}} d t \leq K \varepsilon,
$$

where $K=K(r, k, m)$ depends on the choice of the initial discount factor, the order of integration and the polynomial growth of the coefficients of the state. Let us briefly sketch also the computations for the last addendum

$$
\begin{aligned}
& \int_{0}^{\infty} e^{-r \alpha t}\left(\mathbb{E}\left|D_{x}^{2} \sigma^{j}\left(\bar{X}_{t}, \bar{u}_{t}\right)\left(y_{t}^{\varepsilon}\right)^{2}\right|^{2 k}\right)^{\frac{1}{k}} d t \\
& \leq K\left(\sup _{t \geq 0} e^{-r \alpha k t} \mathbb{E}\left|y_{t}^{\varepsilon}\right|^{8 k}\right)^{\frac{1}{2 k}} \int_{0}^{\infty} e^{-\frac{r \alpha}{2} t}\left(\mathbb{E}\left|D_{x}^{2} \sigma^{j}\left(\bar{X}_{t}, \bar{u}_{t}\right)\right|^{4 k}\right)^{\frac{1}{2 k}} d t \\
& \leq K\left(\sup _{t \geq 0} e^{-r \alpha k t} \mathbb{E}\left|y_{t}^{\varepsilon}\right|^{8 k}\right)^{\frac{1}{2 k}} \int_{0}^{\infty} e^{-\frac{r \alpha}{2} t}\left(\left.\left.\mathbb{E}|1+| \bar{X}_{t}\right|^{m}\right|^{4 k}\right)^{\frac{1}{2 k}} d t
\end{aligned}
$$

If $\alpha \geq 4 \mathrm{~km}$, following the same strategy as above we end up with

$$
\int_{0}^{\infty} e^{-r \alpha t}\left(\mathbb{E}\left|D_{x}^{2} \sigma^{j}\left(\bar{X}_{t}, \bar{u}_{t}\right)\left(y_{t}^{\varepsilon}\right)^{2}\right|^{2 k}\right)^{\frac{1}{k}} d t \leq K \varepsilon^{2} .
$$

Summing up all the estimates and using Lemma 1, we easily get the required result, for some $\rho_{3}$ big enough. In this case it is sufficient to choose $\rho_{3} \geq \alpha r \geq 8 k(2 m+1) r$.

(iv) Following Yong and Zhou [24], it is easy to see that

$$
d \eta_{t}^{\varepsilon}=\left[D_{x} b\left(\bar{X}_{t}, \bar{u}_{t}\right) \eta_{t}^{\varepsilon}+A_{t}^{\varepsilon}\right] d t+\sum_{j=1}^{d}\left[D_{x} \sigma^{j}\left(\bar{X}_{t}, \bar{u}_{t}\right) \eta_{t}^{\varepsilon}+B_{t}^{j, \varepsilon}\right] d W_{t}^{j}
$$

where

$$
\begin{aligned}
A_{t}^{\varepsilon} & :=\delta b_{t} \chi_{E_{\varepsilon}}(t)+\left[G_{b}(t)-D_{x} b\left(\bar{X}_{t}, \bar{u}_{t}\right)\right] \xi_{t}^{\varepsilon} \\
B_{t}^{j, \varepsilon} & :=\left(G_{\sigma}^{j}(t)-D_{x}^{j} \sigma\left(\bar{X}_{t}, \bar{u}_{t}\right)\right) \xi_{t}^{\varepsilon} .
\end{aligned}
$$


Let us consider $A^{\varepsilon}(\cdot)$ first.

$$
\begin{aligned}
\int_{0}^{\infty} e^{-\frac{r \alpha}{2}} t\left(\mathbb{E}\left|A_{t}^{\varepsilon}\right|^{2 k}\right)^{\frac{1}{2 k}} d t \\
\leq K \int_{0}^{\infty} e^{-\frac{r \alpha}{2} t}\left(\mathbb{E}\left|\delta b_{t} \chi_{E_{\varepsilon}}(t)\right|^{2 k}\right)^{\frac{1}{2 k}} d t \\
\left.\quad+\left.K \int_{0}^{\infty} e^{-\frac{r \alpha}{2} t}\left(\mathbb{E} \mid\left[G_{b}(t)-D_{x} b\left(\bar{X}_{t}, \bar{u}_{t}\right)\right)\right] \xi_{t}^{\varepsilon}\right|^{2 k}\right)^{\frac{1}{2 k}} d t \\
\left.\leq K \varepsilon+\left.K\left(\sup _{t \geq 0} e^{-r \alpha k t} \mathbb{E}\left|\xi_{t}^{\varepsilon}\right|^{4 k}\right)^{\frac{1}{4 k}} \int_{0}^{\infty} e^{-\frac{r \alpha}{4} t}\left(\mathbb{E} \mid G_{b}(t)-D_{x} b\left(\bar{X}_{t}, \bar{u}_{t}\right)\right)\right|^{4 k}\right)^{\frac{1}{4 k}} d t \\
\left.\leq K \varepsilon+\left.K \varepsilon^{1 / 2} \int_{0}^{\infty} e^{-\frac{r \alpha}{4} t}\left(\mathbb{E} \mid G_{b}(t)-D_{x} b\left(\bar{X}_{t}, \bar{u}_{t}\right)\right)\right|^{4 k}\right)^{\frac{1}{4 k}} d t,
\end{aligned}
$$

due to the previous result with $\alpha \geq 2$, and Hölder inequality. Regarding the last term we have

$$
\begin{aligned}
\left.G_{b}(t)-D_{x} b\left(\bar{X}_{t}, \bar{u}_{t}\right)\right)= & \left.\int_{0}^{1}\left[D_{x} b\left(\bar{X}_{t}+\theta \xi_{t}^{\varepsilon}, u_{t}^{\varepsilon}\right)-D_{x} b\left(\bar{X}_{t}, \bar{u}_{t}\right)\right)\right] d \theta \\
= & \int_{0}^{1}\left[D_{x} b\left(\bar{X}_{t}+\theta \xi_{t}^{\varepsilon}, u_{t}^{\varepsilon}\right)-D_{x} b\left(\bar{X}_{t}+\theta \xi_{t}^{\varepsilon}, \bar{u}_{t}\right)\right] d \theta \\
& \left.+\int_{0}^{1}\left[D_{x} b\left(\bar{X}_{t}+\theta \xi_{t}^{\varepsilon}, \bar{u}_{t}\right)-D_{x} b\left(\bar{X}_{t}, \bar{u}_{t}\right)\right)\right] d \theta
\end{aligned}
$$

Hence, using the Taylor expansion with Lagrange rest, there exists $\tilde{x}$ (depending on $t$ and $\omega$ ) such that

$$
\begin{aligned}
\int_{0}^{\infty} e^{-\frac{r \alpha}{4} t}\left|G_{b}(t)-D_{x} b\left(\bar{X}_{t}, \bar{u}_{t}\right)\right|_{L^{4 k}(\Omega)} d t \\
\left.\leq\left.\int_{E_{\varepsilon}} e^{-\frac{r \alpha}{4} t}\left(\mathbb{E} \mid \int_{0}^{1} D_{x} b\left(\bar{X}_{t}+\theta \xi_{t}^{\varepsilon}, u_{t}^{\varepsilon}\right)-D_{x} b\left(\bar{X}_{t}+\theta \xi_{t}^{\varepsilon}, \bar{u}_{t}\right)\right] d \theta\right|^{4 k}\right)^{\frac{1}{4 k}} d t \\
\quad+\int_{0}^{\infty} e^{-\frac{r \alpha}{4} t}\left(\mathbb{E}\left|\int_{0}^{1} D_{x}^{2} b\left(\tilde{x}, \bar{u}_{t}\right) \theta \xi^{\varepsilon}(t) d \theta\right|^{4 k}\right)^{\frac{1}{4 k}} d t \\
\leq K \varepsilon+\left(\sup _{t \geq 0} e^{-r \alpha k t} \mathbb{E}\left|\xi_{t}^{\varepsilon}\right|^{8 k}\right)^{\frac{1}{8 k}} \int_{0}^{\infty} e^{-\frac{r \alpha}{8} t}\left(\mathbb{E}\left|D_{x}^{2} b\left(\tilde{x}, \bar{u}_{t}\right)\right|^{8 k}\right)^{\frac{1}{8 k}} d t \\
\leq K\left(\varepsilon+\varepsilon^{1 / 2}\right),
\end{aligned}
$$

thanks to the estimate obtained in point (i) and the polynomial growth of $D_{x}^{2} b$ (here we have to require $\alpha \geq 32 k(2 m+1))$. Then

$$
\begin{aligned}
\int_{0}^{\infty} e^{-\frac{r \alpha}{2} t}\left(\mathbb{E}\left|A_{t}^{\varepsilon}\right|^{2 k}\right)^{\frac{1}{2 k}} d t & \leq K \varepsilon+K \varepsilon^{1 / 2} \int_{0}^{\infty} e^{-\frac{r \alpha}{4} t}\left(\mathbb{E}\left|G_{b}(t)-D_{x} b\left(\bar{X}_{t}, \bar{u}_{t}\right)\right|^{4 k}\right)^{\frac{1}{4 k}} d t \\
& \leq K \varepsilon .
\end{aligned}
$$

For $B_{t}^{j, \varepsilon}$, proceeding in a similar way we obtain

$$
\int_{0}^{\infty} e^{-r \alpha t}\left(\mathbb{E}\left|G_{\sigma}^{j}(t)-D_{x} \sigma^{j}\left(\bar{X}_{t}, \bar{u}_{t}\right)\right|^{4 k}\right)^{\frac{1}{2 k}} d t \leq K \varepsilon .
$$

To conclude, we apply Lemma 1 to get

$$
\begin{aligned}
\sup _{t \in \mathbb{R}_{+}} e^{-r \alpha k t} \mathbb{E}\left|\eta_{t}^{\varepsilon}\right|^{2 k} & \leq K\left(\int_{0}^{\infty} e^{-\frac{r \alpha}{2} t}\left(\mathbb{E}\left|A_{t}^{\varepsilon}\right|^{2 k}\right)^{\frac{1}{2 k}} d t\right)^{2 k}+K \sum_{j=1}^{d}\left(\int_{0}^{\infty} e^{-r \alpha t}\left(\mathbb{E}\left|B_{t}^{j, \varepsilon}\right|^{2 k}\right)^{\frac{1}{k}} d t\right)^{k} \\
& \leq K\left(\varepsilon^{2 k}+\varepsilon^{2 k}\right)=O\left(\varepsilon^{2 k}\right) .
\end{aligned}
$$

In this case $\rho_{4}$ can be chosen as $\rho_{4} \geq r \alpha \geq 32 k(2 m+1) r$. 
(v) Let us denote $d \zeta^{\varepsilon}(t)=d\left(\eta^{\varepsilon}(t)-\xi^{\varepsilon}(t)\right)$,

$$
\left\{\begin{array}{l}
d \zeta_{t}^{\varepsilon}=\left(D_{x} b\left(\bar{X}_{t}, u_{t}\right) \zeta_{t}^{\varepsilon}+A_{t}^{\varepsilon}\right) d t+\sum_{j=1}^{d}\left(D_{x} \sigma^{j}\left(\bar{X}_{t}, u_{t}\right) \zeta_{t}^{\varepsilon}+B_{t}^{j, \varepsilon}\right) d W_{t}^{j}, \\
\zeta^{\varepsilon}(0)=0
\end{array}\right.
$$

where

$$
\begin{aligned}
A_{T}^{\varepsilon}:= & \delta D_{x} b_{t} \chi_{E_{\varepsilon}}(t) \xi_{t}^{\varepsilon}+\frac{1}{2}\left[\widetilde{G}_{b}(t)-D_{x}^{2} b\left(\bar{X}_{t}, u_{t}^{\varepsilon}\right)\right]\left(\xi_{t}^{\varepsilon}\right)^{2} \\
& +\frac{1}{2} \delta D_{x}^{2} b_{t} \chi_{E_{\varepsilon}}(t)\left(\xi_{t}^{\varepsilon}\right)^{2}+\frac{1}{2} D_{x}^{2} b\left(\bar{X}_{t}, u_{t}\right)\left[\left(\xi_{t}^{\varepsilon}\right)^{2}-\left(y_{t}^{\varepsilon}\right)^{2}\right], \\
B_{t}^{\varepsilon}:= & \delta D_{x} \sigma_{t} \chi_{E_{\varepsilon}}(t) \eta_{t}^{\varepsilon}+\frac{1}{2}\left[\widetilde{G}_{\sigma}(t)-D_{x}^{2} \sigma\left(\bar{X}_{t}, u_{t}^{\varepsilon}\right)\right]\left(\xi_{t}^{\varepsilon}\right)^{2} \\
& +\frac{1}{2} \delta D_{x}^{2} \sigma_{t} \chi_{E_{\varepsilon}}(t)\left(\xi_{t}^{\varepsilon}\right)^{2}+\frac{1}{2} D_{x}^{2} \sigma\left(\bar{X}_{t}, u_{t}\right)\left[\left(\xi_{t}^{\varepsilon}\right)^{2}-\left(y_{t}^{\varepsilon}\right)^{2}\right],
\end{aligned}
$$

and

$$
\left\{\begin{array}{l}
\widetilde{G}_{b}(t):=2 \int_{0}^{1} \theta D_{x}^{2} b\left(\theta \bar{X}_{t}+(1-\theta) X_{t}^{\varepsilon}, u_{t}^{\varepsilon}\right) d \theta \\
\widetilde{G}_{\sigma}(t):=2 \int_{0}^{1} \theta D_{x}^{2} \sigma\left(\theta \bar{X}_{t}+(1-\theta) X_{t}^{\varepsilon}, u_{t}^{\varepsilon}\right) d \theta
\end{array}\right.
$$

First, let us consider the $A^{\varepsilon}(\cdot)$ term. Applying the Hölder inequality gives

$$
\begin{aligned}
& \int_{0}^{\infty} e^{-\frac{r \alpha}{2} t}\left(\mathbb{E}\left|A_{t}^{\varepsilon}\right|^{2 k}\right)^{\frac{1}{2 k}} d t \\
& \leq \int_{0}^{\infty} e^{-\frac{r}{2} t}\left[\left(\mathbb{E}\left|\delta D_{x} b_{t} \chi_{E_{\varepsilon}}(t) \xi_{t}^{\varepsilon}\right|^{2 k}\right)^{\frac{1}{2 k}}+\frac{1}{2}\left(\mathbb{E}\left|\left[\widetilde{G}_{b}(t)-D_{x}^{2} b\left(\bar{X}(t), u_{t}^{\varepsilon}\right)\right]\left(\xi_{t}^{\varepsilon}\right)^{2}\right|^{2 k}\right)^{\frac{1}{2 k}}\right. \\
&\left.+\frac{1}{2}\left(\mathbb{E}\left|\delta D_{x}^{2} b_{t} \chi_{E_{\varepsilon}}(t)\left(\xi_{t}^{\varepsilon}\right)^{2}\right|^{2 k}\right)^{\frac{1}{2 k}}+\frac{1}{2}\left(\mathbb{E}\left|D_{x}^{2} b\left(\bar{X}_{t}, u_{t}\right)\left[\left(\xi_{t}^{\varepsilon}\right)^{2}-\left(y_{t}^{\varepsilon}\right)^{2}\right]\right|^{2 k}\right)^{\frac{1}{2 k}}\right] d t \\
& \leq K\left(\sup _{t \geq 0} e^{-r \alpha k t} \mathbb{E}\left|\xi_{t}^{\varepsilon}\right|^{4 k}\right)^{\frac{1}{4 k}} \int_{E_{\varepsilon}} e^{-\frac{r \alpha}{4} t}\left(\mathbb{E}\left|\delta D_{x} b_{t}\right|^{4 k}\right)^{\frac{1}{4 k}} d t \\
&+K\left(\sup _{t \geq 0} e^{-r \alpha k t} \mathbb{E}\left|\xi_{t}^{\varepsilon}\right|^{8 k}\right)^{\frac{1}{4 k}} \int_{0}^{\infty} e^{-\frac{r \alpha}{4} t}\left(\mathbb{E}\left|\widetilde{G}_{b}(t)-D_{x}^{2} b\left(\bar{X}_{t}, u_{t}^{\varepsilon}\right)\right|^{4 k}\right)^{\frac{1}{4 k}} d t \\
&+K\left(\sup _{t \geq 0} e^{-r \alpha k t} \mathbb{E}\left|\xi_{t}^{\varepsilon}\right|^{8 k}\right)^{\frac{1}{4 k}} \int_{E_{\varepsilon}} e^{-\frac{r \alpha}{4} t}\left(\mathbb{E}\left|\delta D_{x}^{2} b_{t}\right|^{4 k}\right)^{\frac{1}{4 k}} d t \\
&+K\left(\sup _{t \geq 0} e^{-r \alpha k t} \mathbb{E}\left|\eta_{t}^{\varepsilon}\right|^{8 k}\right)^{\frac{1}{8 k}}\left(\sup _{t \geq 0} e^{-r \alpha k t} \mathbb{E}\left|\xi_{t}^{\varepsilon}+y_{t}^{\varepsilon}\right|^{8 k}\right)^{\frac{1}{8 k}} \int_{0}^{\infty} e^{-\frac{r \alpha t}{4}}\left(\mathbb{E}\left|D_{x}^{2} b\left(\bar{X}_{t}, u_{t}\right)\right|^{4 k}\right)^{\frac{1}{4 k}} d t .
\end{aligned}
$$

If $\alpha \geq 4$ the first and the third term con be easily controlled. For the last addendum we use the same technique as in (iii) to get the boundedness of the integral for $\alpha \geq 8 k(2 m+1)$, hence

$$
\int_{0}^{\infty} e^{-\frac{r \alpha}{2} t}\left(\mathbb{E}\left|A_{t}^{\varepsilon}\right|^{2 k}\right)^{\frac{1}{2 k}} d t \leq K\left[\varepsilon^{3 / 2}+\varepsilon \int_{0}^{\infty} e^{-\frac{r \alpha}{4} t}\left(\mathbb{E}\left|\widetilde{G}_{b}(t)-D_{x}^{2} b\left(\bar{X}_{t}, u_{t}^{\varepsilon}\right)\right|^{4 k}\right)^{\frac{1}{4 k}} d t+\varepsilon^{2}+\varepsilon^{3 / 2}\right] .
$$

Finally, we can rewrite $\mathbb{E}\left|\widetilde{G}_{b}(t)-D_{x}^{2} b\left(t, \bar{x}(t), u^{\varepsilon}(t)\right)\right|^{4 k}$ in the following form

$$
\begin{aligned}
& \widetilde{G}_{b}(t)-D_{x}^{2} b\left(\bar{X}_{t}, u_{t}^{\varepsilon}\right)= \\
& =2 \int_{0}^{1} \theta D_{x}^{2} b\left(\theta \bar{X}_{t}+(1-\theta) X_{t}^{\varepsilon}, u_{t}^{\varepsilon}\right) d \theta-D_{x}^{2} b\left(\bar{X}_{t}, u_{t}^{\varepsilon}\right) \\
& =2 \int_{0}^{1} \theta\left[D_{x}^{2} b\left(\theta \bar{X}_{t}+(1-\theta) X_{t}^{\varepsilon}, u_{t}^{\varepsilon}\right)-D_{x}^{2} b\left(\bar{X}_{t}, u_{t}^{\varepsilon}\right)\right] d \theta .
\end{aligned}
$$

If $\alpha \geq 8 k(2 m+1)$, by the continuity of the map $x \mapsto D_{x}^{2} b(x, u)$ and dominated convergence theorem it follows that

$$
\int_{0}^{\infty} e^{-\frac{r \alpha}{4} t}\left(\int_{0}^{1} \theta\left[D_{x}^{2} b\left(\theta \bar{X}_{t}+(1-\theta) X_{t}^{\varepsilon}, u_{t}^{\varepsilon}\right)-D_{x}^{2} b\left(\bar{X}_{t}, u_{t}^{\varepsilon}\right)\right] d \theta\right)^{\frac{1}{4 k}} d t \rightarrow 0
$$


as $\varepsilon \rightarrow 0$. Then

$$
\int_{0}^{\infty} e^{-\frac{r \alpha}{2} t}\left(\mathbb{E}\left|A_{t}^{\varepsilon}\right|^{2 k}\right)^{\frac{1}{2 k}} d t \leq K\left[\varepsilon^{3 / 2}+\varepsilon^{2}+\varepsilon^{3 / 2}\right]+o(\varepsilon)=o(\varepsilon)
$$

For $B^{\varepsilon}(t)$ we proceed in the same way to obtain

$$
\int_{0}^{\infty} e^{-\frac{r t}{2 k}}\left(\mathbb{E}\left|B^{j, \varepsilon}(t)\right|^{2 k}\right)^{\frac{1}{k}} d t=o\left(\varepsilon^{2}\right)
$$

Using Lemma 1, the desired result follows for $\rho_{5} \geq r \alpha \geq 8 k(2 m+1) r$.

\subsection{Proof of Proposition 3 .}

Proof. Since $Y_{t}^{\varepsilon}=y_{t}^{\varepsilon}\left(y_{t}^{\varepsilon}\right)^{T}$, the existence and uniqueness of a solution follow from the existence and uniqueness of the process $y^{\varepsilon}$ (see Theorem 2), with the restriction $r>2 c_{1 / 2}$.

Let us now denote $A_{t}:=D_{x} b\left(\bar{X}_{t}, \bar{u}_{t}\right), B_{t}^{j}:=D_{x} \sigma^{j}\left(\bar{X}_{t}, \bar{u}_{t}\right)$ and note that a symmetric (positive) definite matrix $Y_{t}^{\varepsilon}$ can be decomposed as $Y_{t}^{\varepsilon}=\sum_{i=1}^{n} \gamma_{i} c_{i} c_{i}^{T}$, where $\gamma_{i} \geq 0$ for all $i$ and $\left(c_{i}\right)_{i}$ is an orthonormal basis of $\mathbb{R}^{n}$. Clearly, each $\gamma_{i}$ and $c_{i}$ depend both on $t$ and $\varepsilon$ but we omit this notation in the proof.

Having in mind the above, one arrives at

$$
\begin{gathered}
\left\langle A_{t} Y_{t}^{\varepsilon}, Y_{t}^{\varepsilon}\right\rangle_{2}=\operatorname{Tr}\left\{A_{t} Y_{t}^{\varepsilon}\left(Y_{t}^{\varepsilon}\right)^{T}\right\}=\sum_{i=1}^{n} \gamma_{i}^{2} \operatorname{Tr}\left\{A_{t} c_{i} c_{i}^{T} c_{i} c_{i}^{T}\right\}=\sum_{i=1}^{n} \gamma_{i}^{2} \operatorname{Tr}\left\{c_{i} c_{i}^{T} A_{t} c_{i} c_{i}^{T}\right\} \\
=\sum_{i=1}^{n} \gamma_{i}^{2}\left\langle A_{t} c_{i}, c_{i}\right\rangle \operatorname{Tr}\left\{c_{i} c_{i}^{T}\right\}=\sum_{i=1}^{n} \gamma_{i}^{2}\left\langle A_{t} c_{i}, c_{i}\right\rangle \\
\left\langle B_{t}^{j} Y_{t}^{\varepsilon}\left(B_{t}^{j}\right)^{T}, Y_{t}^{\varepsilon}\right\rangle_{2}=\operatorname{Tr}\left\{B_{t}^{j} Y_{t}^{\varepsilon}\left(B_{t}^{j}\right)^{T}\left(Y_{t}^{\varepsilon}\right)^{T}\right\}=\sum_{i=1}^{n} \gamma_{i}^{2} \operatorname{Tr}\left\{B_{t}^{j} c_{i}\left(c_{i}^{T}\left(B_{t}^{j}\right)^{T} c_{i}\right) c_{i}^{T}\right\} \\
=\sum_{i=1}^{n} \gamma_{i}^{2}\left\langle\left(B_{t}^{j}\right)^{T} c_{i}, c_{i}\right\rangle^{2} \leq \sum_{i=1}^{n}\left|\left(B_{t}^{j}\right)^{T}\left(\gamma_{i} c_{i}\right)\right|^{2}\left|c_{i}\right|^{2} \\
=\sum_{i=1}^{n}\left|\left(B_{t}^{j}\right)^{T}\left(\gamma_{i} c_{i}\right)\right|^{2}=\sum_{i=1}^{n} \gamma_{i}^{2}\left|\left(B_{t}^{j}\right)^{T} c_{i}\right|^{2},
\end{gathered}
$$

and

$$
\begin{aligned}
\left\|B_{t}^{j} Y_{t}^{\varepsilon}\right\|_{2}^{2} & =\operatorname{Tr}\left\{B_{t}^{j} Y_{t}^{\varepsilon}\left(Y_{t}^{\varepsilon}\right)^{T}\left(B_{t}^{j}\right)^{T}\right\}=\sum_{i=1}^{n} \gamma_{i}^{2} \operatorname{Tr}\left\{B_{t}^{j} c_{i} c_{i}^{T} c_{i} c_{i}^{T}\left(B_{t}^{j}\right)^{T}\right\} \\
& =\sum_{i=1}^{n} \gamma_{i}^{2} \operatorname{Tr}\left\{c_{i} c_{i}^{T}\left(B_{t}^{j}\right)^{T} B_{t}^{j} c_{i} c_{i}^{T}\right\}=\sum_{i=1}^{n} \gamma_{i}^{2}\left\langle\left(B_{t}^{j}\right)^{T} B_{t}^{j} c_{i}, c_{i}\right\rangle \\
& =\sum_{i=1}^{n} \gamma_{i}^{2}\left|B_{t}^{j} c_{i}\right|^{2},
\end{aligned}
$$

where we have used the basic properties of the Trace. Using these estimates we are able to prove the following dissipativity condition

$$
\begin{aligned}
& \left\langle A_{t} Y_{t}^{\varepsilon}+Y_{t}^{\varepsilon} A_{t}^{T}, Y_{t}^{\varepsilon}\right\rangle+\sum_{j=1}^{d}\left\langle B_{t}^{j} Y_{t}^{\varepsilon}\left(B_{t}^{j}\right)^{T}, Y_{t}^{\varepsilon}\right\rangle+\sum_{j=1}^{d}\left\|B_{t}^{j} Y_{t}^{\varepsilon}+Y_{t}^{\varepsilon} B_{t}^{j}\right\|_{2}^{2} \\
& \leq 2 \sum_{i=1}^{n} \gamma_{i}^{2}\left\langle A_{t} c_{i}, c_{i}\right\rangle+\sum_{j=1}^{d} \sum_{i=1}^{n} \gamma_{i}^{2}\left|\left(B_{t}^{j}\right)^{T} c_{i}\right|^{2}+2 \sum_{j=1}^{d} \sum_{i=1}^{n} \gamma_{i}^{2}\left|B_{t}^{j} c_{i}\right|^{2} \\
& =2 \sum_{i=1}^{n} \gamma_{i}^{2}\left(\left\langle A_{t} c_{i}, c_{i}\right\rangle+\frac{3}{2} \sum_{j=1}^{d}\left|B_{t}^{j} c_{i}\right|^{2}\right) \\
& \leq 2 c_{3 / 2} \sum_{i=1}^{n} \gamma_{i}^{2}\left|c_{i}\right|^{2}=2 c_{3 / 2}\left\|Y_{t}^{\varepsilon}\right\|_{2}^{2} .
\end{aligned}
$$


Now, applying the Itô formula to $e^{-r t}\left\|Y_{t}^{\varepsilon}\right\|_{2}^{2}$ we obtain, for every $T>0$ and $\delta>0$

$$
\begin{aligned}
& \mathbb{E}\left[e^{-r t}\left\|Y_{t}^{\varepsilon}\right\|_{2}^{2}\right]+r \mathbb{E} \int_{0}^{T} e^{-r t}\left\|Y_{t}^{\varepsilon}\right\|_{2}^{2} d t \\
&=2 \mathbb{E} \int_{0}^{T} e^{-r t}\left[\left\langle Y_{t}^{\varepsilon}, A_{t} Y_{t}^{\varepsilon}+Y_{t}^{\varepsilon} A_{t}^{T}\right\rangle+\sum_{j=1}^{d}\left\langle Y_{t}^{\varepsilon}, B_{t}^{j} Y_{t}^{\varepsilon}\left(B_{t}^{j}\right)^{T}\right\rangle\right] d t \\
& \quad+2 \mathbb{E} \int_{0}^{T} e^{-r t}\left\langle Y_{t}^{\varepsilon}, \Gamma_{t}\right\rangle d t+\sum_{j=1}^{d} \mathbb{E} \int_{0}^{T} e^{-r t}\left\|B_{t}^{j} Y_{t}^{\varepsilon}+Y_{t}^{\varepsilon} B_{t}^{j}+\Lambda_{t}^{j}\right\|_{2}^{2} d t \\
& \leq 2 c_{3 / 2} \mathbb{E} \int_{0}^{T} e^{-r t}\left\|Y_{t}^{\varepsilon}\right\|_{2}^{2} d t+\delta \mathbb{E} \int_{0}^{T} e^{-r t}\left\|Y_{t}^{\varepsilon}\right\|_{2}^{2} d t \\
&+\frac{1}{\delta} \mathbb{E} \int_{0}^{T} e^{-r t}\left\|\Gamma_{t}\right\|_{2}^{2} d t+\sum_{j=1}^{d} \int_{0}^{T} e^{-r t}\left\|\Lambda_{t}^{j}\right\|_{2}^{2} d t .
\end{aligned}
$$

Hence

$$
\left(r-2 c_{3 / 2}-\delta\right) \mathbb{E} \int_{0}^{T} e^{-r t}\left\|Y_{t}^{\varepsilon}\right\|_{2}^{2} d t \leq \frac{1}{\delta} \mathbb{E} \int_{0}^{T} e^{-r t}\left\|\Gamma_{t}\right\|_{2}^{2} d t+\sum_{j=1}^{d} \int_{0}^{T} e^{-r t}\left\|\Lambda_{t}^{j}\right\|_{2}^{2} d t,
$$

and the estimate follows for $r>2 c_{3 / 2}$ by sending $T \rightarrow+\infty$. The final estimate holds for $r>$ $2 \max \left\{c_{1 / 2}, c_{3 / 2}\right\}$.

\subsection{Proof of Proposition 7 .}

Proof. If we compute the Itô differential of the processes $e^{-r t}\left\langle y_{t}^{\varepsilon}, p_{t}\right\rangle$, where $p_{t}$ is the solution to the finite horizon equation (37), we obtain

$$
\begin{aligned}
d\left(e^{-r t}\left\langle y_{t}^{\varepsilon}, p_{t}\right\rangle\right)= & \left\langle d\left(e^{-r t} y_{t}^{\varepsilon}\right), p_{t}\right\rangle+e^{-r t}\left\langle y_{t}, d p_{t}\right\rangle+\sum_{j=1}^{d}\left\langle q_{t}^{j}, D_{x} \sigma^{j}\left(\bar{X}_{t}, \bar{u}_{t}\right) y_{t}^{\varepsilon}+\delta \sigma^{j}\left(\bar{X}_{t}, \bar{u}_{t}\right)\right\rangle d t \\
= & {\left[-r e^{-r t}\left\langle y_{t}^{\varepsilon}, p_{t}\right\rangle+e^{-r t}\left\langle D_{x} b\left(\bar{X}_{t}, \bar{u}_{t}\right) y_{t}^{\varepsilon}, p_{t}\right\rangle-e^{-r t}\left\langle y_{t}^{\varepsilon}, D_{x} b\left(\bar{X}_{t}, \bar{u}_{t}\right)^{T} y_{t}^{\varepsilon}\right\rangle\right.} \\
& -\sum_{j=1}^{d} e^{-r t}\left\langle y_{t}^{\varepsilon}, D_{x} \sigma^{j}\left(\bar{X}_{t}, \bar{u}_{t}\right)^{T} q_{t}^{j}\right\rangle+e^{-r t}\left\langle y_{t}^{\varepsilon}, D_{x} f\left(\bar{X}_{t}, \bar{u}_{t}\right)\right\rangle+r e^{-r t}\left\langle y_{t}^{\varepsilon}, p_{t}\right\rangle \\
& \left.+\sum_{j=1}^{d}\left\langle q_{t}^{j}, D_{x} \sigma^{j}\left(\bar{X}_{t}, \bar{u}_{t}\right) y_{t}^{\varepsilon}+\delta \sigma^{j}\left(\bar{X}_{t}, \bar{u}_{t}\right)\right\rangle\right] d t+M_{t} d W_{t},
\end{aligned}
$$

where the stochastic term is a local martingale with zero mean value (which can be proved by standard localization argument). Hence, by taking expectation we have for all $T>0$

$$
e^{-r T} \mathbb{E}\left\langle y_{T}^{\varepsilon}, p_{T}\right\rangle-\mathbb{E} \int_{0}^{T} e^{-r t}\left\langle y_{t}^{\varepsilon}, D_{x} f\left(\bar{X}_{t}, \bar{u}_{t}\right)\right\rangle d t=\sum_{j=1}^{d} \mathbb{E} \int_{0}^{T} e^{-r t}\left\langle q_{t}^{j}, \delta \sigma^{j}\left(\bar{X}_{t}, \bar{u}_{t}\right)\right\rangle d t,
$$

thanks to the fact that $y_{0}^{\varepsilon}=0$. Since $\left(p_{t}\right)_{t \geq 0} \in L_{\mathcal{F}}^{2,-r}\left(\mathbb{R}_{+} ; \mathbb{R}^{n}\right)$ then there exists a sequence of times $\left(T_{n}\right)_{n \geq 1}$ with $T_{n} \nearrow+\infty$ as $n \rightarrow+\infty$ such that along this sequence $\mathbb{E}\left[e^{-r T_{n}} p_{T_{n}}\right] \rightarrow 0$. Hence, for all $n \in \mathbb{N}$ we have that

$$
\mathbb{E}\left\langle e^{-r T_{n}} y_{T_{n}}^{\varepsilon}, p_{T_{n}}\right\rangle-\mathbb{E} \int_{0}^{T_{n}} e^{-r t}\left\langle y_{t}^{\varepsilon}, D_{x} f\left(\bar{X}_{t}, \bar{u}_{t}\right)\right\rangle d t=\sum_{j=1}^{d} \mathbb{E} \int_{0}^{T_{n}} e^{-r t}\left\langle q_{t}^{j}, \delta \sigma^{j}\left(\bar{X}_{t}, \bar{u}_{t}\right)\right\rangle d t .
$$

Thanks to the growth assumptions on $\sigma, f$ and to the regularity of $y_{t}^{\varepsilon}$ and $q_{t}$, we can send $T_{n}$ to infinity to end with

$$
\mathbb{E} \int_{0}^{\infty} e^{-r t}\left\langle y_{t}^{\varepsilon}, D_{x} f\left(\bar{X}_{t}, \bar{u}_{t}\right)\right\rangle d t=-\sum_{j=1}^{d} \mathbb{E} \int_{0}^{\infty} e^{-r t}\left\langle q_{t}^{j}, \delta \sigma^{j}\left(\bar{X}_{t}, \bar{u}_{t}\right)\right\rangle d t
$$


Repeating the same argument for $e^{-r t}\left\langle z_{t}^{\varepsilon}, p_{t}\right\rangle$ we get

$$
\begin{aligned}
d\left(e^{-r t}\left\langle z_{t}^{\varepsilon}, p_{t}\right\rangle\right)= & \left\langle d\left(e^{-r t} z_{t}^{\varepsilon}\right), p_{t}\right\rangle+e^{-r t}\left\langle z_{t}, d p_{t}\right\rangle \\
& +\sum_{j=1}^{d}\left\langle q_{t}^{j}, D_{x} \sigma^{j}\left(\bar{X}_{t}, \bar{u}_{t}\right) z_{t}^{\varepsilon}+\delta \sigma^{j}\left(\bar{X}_{t}, \bar{u}_{t}\right) y_{t}^{\varepsilon}+\frac{1}{2} D_{x}^{2} \sigma^{j}\left(y_{t}^{\varepsilon}\right)^{2}\right\rangle d t \\
= & {\left[-r e^{-r t}\left\langle z_{t}^{\varepsilon}, p_{t}\right\rangle+e^{-r t}\left\langle D_{x} b\left(\bar{X}_{t}, \bar{u}_{t}\right) z_{t}^{\varepsilon}, p_{t}\right\rangle+e^{-r t}\left\langle\delta b\left(\bar{X}_{t}, \bar{u}_{t}\right), p_{t}\right\rangle\right.} \\
& +\frac{1}{2} e^{-r t}\left\langle D_{x}^{2} b\left(\bar{X}_{t}, \bar{u}_{t}\right)\left(y_{t}^{\varepsilon}\right)^{2}, p_{t}\right\rangle-e^{-r t}\left\langle z_{t}^{\varepsilon}, D_{x} b\left(\bar{X}_{t}, \bar{u}_{t}\right)^{T} y_{t}^{\varepsilon}\right\rangle \\
& -\sum_{j=1}^{d} e^{-r t}\left\langle z_{t}^{\varepsilon}, D_{x} \sigma^{j}\left(\bar{X}_{t}, \bar{u}_{t}\right)^{T} q_{t}^{j}\right\rangle+e^{-r t}\left\langle z_{t}^{\varepsilon}, D_{x} f\left(\bar{X}_{t}, \bar{u}_{t}\right)\right\rangle \\
& +r e^{-r t}\left\langle z_{t}^{\varepsilon}, p_{t}\right\rangle+\sum_{j=1}^{d}\left\langle q_{t}^{j}, D_{x} \sigma^{j}\left(\bar{X}_{t}, \bar{u}_{t}\right) z_{t}^{\varepsilon}\right\rangle \\
& \left.+\sum_{j=1}^{d}\left\langle q_{t}^{j}, \delta \sigma^{j}\left(\bar{X}_{t}, \bar{u}_{t}\right) y_{t}^{\varepsilon}+\frac{1}{2} D_{x}^{2} \sigma^{j}\left(y_{t}^{\varepsilon}\right)^{2}\right\rangle\right] d t+N_{t} d W_{t},
\end{aligned}
$$

where the stochastic term is a local martingale with zero mean value (which can be proved by same argument as before). Hence, taking expectation we obtain for all $T>0$

$$
\begin{aligned}
-\mathbb{E} \int_{0}^{T} e^{-r t}\left\langle z_{t}^{\varepsilon}, D_{x} f\left(\bar{X}_{t}, \bar{u}_{t}\right)\right\rangle d t= & \mathbb{E} \int_{0}^{T} e^{-r t}\left\langle\delta b\left(\bar{X}_{t}, \bar{u}_{t}\right)+\frac{1}{2} D_{x}^{2} b\left(\bar{X}_{t}, \bar{u}_{t}\right)\left(y_{t}^{\varepsilon}\right)^{2}, p_{t}\right\rangle d t \\
& +\frac{1}{2} \sum_{j=1}^{d} \mathbb{E} \int_{0}^{T} e^{-r t}\left\langle D_{x}^{2} \sigma^{j}\left(\bar{X}_{t}, \bar{u}_{t}\right)\left(y_{t}^{\varepsilon}\right)^{2}, q_{t}\right\rangle d t+o(\varepsilon) .
\end{aligned}
$$

The term $o(\varepsilon)$ comes from the following estimate

$$
\begin{aligned}
& \left|\mathbb{E} \int_{0}^{\infty} e^{-r t}\left\langle\delta D_{x} \sigma^{j}\left(\bar{X}_{t}, \bar{u}_{t}\right) y_{t}^{\varepsilon}, q_{t}^{j}\right\rangle d t\right| \\
& \quad \leq C \mathbb{E} \int_{E_{\varepsilon}} e^{-r t}\left(1+\left|\bar{X}_{t}\right|^{m}\right)\left|y_{t}^{\varepsilon}\right|\left|q_{t}^{j}\right| d t \\
& \quad \leq C \int_{E_{\varepsilon}}\left(\mathbb{E}\left[e^{-r t}\left(1+\left|\bar{X}_{t}\right|^{m}\right)^{2}\left|y_{t}^{\varepsilon}\right|^{2}\right]\right)^{1 / 2}\left(\mathbb{E} e^{-r t}\left|q_{t}^{j}\right|^{2}\right)^{1 / 2} d t \\
& \quad \leq C \sup _{t \in E_{\varepsilon}}\left(\mathbb{E} e^{-r t}\left|y_{t}^{\varepsilon}\right|^{4}\right)^{1 / 4} \int_{E_{\varepsilon}}\left(\mathbb{E} e^{-r t}\left|q_{t}^{j}\right|^{2}\right)^{1 / 2} d t \\
& \quad \leq C \varepsilon\left(\int_{E_{\varepsilon}} \mathbb{E} e^{-r t}\left|q_{t}^{j}\right|^{2} d t\right)^{1 / 2},
\end{aligned}
$$

and the last integral goes to zero as $\varepsilon$ goes to zero, since $\mathbb{E} \int_{0}^{\infty} e^{-r t}\left|q_{t}^{j}\right|^{2} d t<\infty$. Applying the same strategy as before we can choose a sequence $\left(T_{n}\right)_{n \geq 1}$ with $T_{n} \nearrow+\infty$ as $n \rightarrow+\infty$ such that along this sequence $\mathbb{E}\left[e^{-r T_{n}} p_{T_{n}}\right] \rightarrow 0$. This way we end up with

$$
\begin{aligned}
-\mathbb{E} \int_{0}^{\infty} e^{-r t}\left\langle z_{t}^{\varepsilon}, D_{x} f\left(\bar{X}_{t}, \bar{u}_{t}\right)\right\rangle d t & \\
= & \mathbb{E} \int_{0}^{\infty} e^{-r t}\left\langle\delta b\left(\bar{X}_{t}, \bar{u}_{t}\right)+\frac{1}{2} D_{x}^{2} b\left(\bar{X}_{t}, \bar{u}_{t}\right)\left(y_{t}^{\varepsilon}\right)^{2}, p_{t}\right\rangle d t \\
& +\frac{1}{2} \sum_{j=1}^{d} \mathbb{E} \int_{0}^{\infty} e^{-r t}\left\langle D_{x}^{2} \sigma^{j}\left(\bar{X}_{t}, \bar{u}_{t}\right)\left(y_{t}^{\varepsilon}\right)^{2}, q_{t}\right\rangle d t+o(\varepsilon) .
\end{aligned}
$$


If we substitute relations (82) and (83) into equation (33), we obtain

$$
\begin{aligned}
J\left(u^{\varepsilon}(\cdot)\right)-J(\bar{u}(\cdot))= & \mathbb{E} \int_{0}^{\infty} e^{-r t}\left[-\sum_{j=1}^{d}\left\langle q_{t}^{j}, \delta \sigma^{j}\left(\bar{X}_{t}, \bar{u}_{t}\right)\right\rangle-\left\langle p_{t}, \delta b\left(\bar{X}_{t}, \bar{u}_{t}\right)\right\rangle+\delta f\left(\bar{X}_{t}, \bar{u}_{t}\right)\right] d t \\
& +\frac{1}{2} \mathbb{E} \int_{0}^{\infty} e^{-r t}\left[-\sum_{j=1}^{d}\left\langle D_{x}^{2} \sigma^{j}\left(\bar{X}_{t}, \bar{u}_{t}\right)\left(y_{t}^{\varepsilon}\right)^{2}, q_{t}\right\rangle-\left\langle D_{x}^{2} b\left(\bar{X}_{t}, \bar{u}_{t}\right)\left(y_{t}^{\varepsilon}\right)^{2}, p_{t}\right\rangle\right. \\
& \left.+\left\langle D_{x}^{2} f\left(\bar{X}_{t}, \bar{u}_{t}\right) y_{t}, y_{t}\right\rangle\right] d t+o(\varepsilon),
\end{aligned}
$$

and recalling the definition of the Hamiltonian $H(x, u, p, q)=\langle p, b(x, u)\rangle+\operatorname{Tr}\left[q^{T} \sigma(x, u)\right]-f(x, u)$ we have the desired result.

10.4. Conditions on the discount factor $r$. Here we collect some restrictions on the discount factor used throughout the computations in the paper. For the purposes of the SMP it is not necessary to find precise values of the discount factor, in general $r$ has to be positive and big enough. Nevertheless, it can be useful to exhibit some sufficient conditions.

Starting from the well posedness of the state equation, we have to require $r>2 c_{1 / 2}$ in order to find a unique solution in the space $L_{\mathcal{F}}^{2,-r}\left(\mathbb{R}_{+} ; \mathbb{R}^{n}\right)$. Regarding the first variation equation we have no other restriction. On the contrary, to assure that $D_{x}^{2} b\left(\bar{X}_{t}, \bar{u}_{t}\right)\left(y_{t}^{\varepsilon}\right)^{2}, D_{x}^{2} \sigma^{j}\left(\bar{X}_{t}, \bar{u}_{t}\right)\left(y_{t}^{\varepsilon}\right)^{2} \in$ $L_{\mathcal{F}}^{2,-r}\left(\mathbb{R}_{+} ; \mathbb{R}^{n}\right)$ in the equation for $z^{\varepsilon}$, a sufficient condition is $r>2 \max \left\{0, c_{1 / 2}, c_{2(2 m+1)-1}, c_{2 m-1}, c_{3}\right\}$, where $c_{3}$ comes from estimate (18) applied to the process $y^{\varepsilon}$. Further restrictions come from the proof of Proposition 1. Here it follows that one can choose $\rho_{1}, \rho_{2} \geq 2 c_{1 / 2} ; \rho_{3}, \rho_{5} \geq 16 k(2 m+$ 1) $\max \left\{c_{2 k(2 m+1)-1}, c_{8 k-1}, c_{2 k m-1}\right\}$ and $\rho_{4} \geq 64 k(2 m+1) \max \left\{c_{4 k(2 m+1)-1}, c_{4 k m-1}\right\}$. These conditions are derived from the polynomial growth assumptions and from the use of the Hölder inequality.

The choice of the discount factor $r$ for the first adjoint equation (see Theorem 4) depends on the a priori estimate given by Lemma 3 as well as the integrability of the forcing term $D_{x} f\left(\bar{X}_{t}, \bar{u}_{t}\right)$. Therefore, due to the polynomial growth, it is easy to see that it is sufficient to consider $r>$ $2 \max \left\{0, c_{1 / 2}, c_{l-1}\right\}$.

For the existence and uniqueness of $\left(y_{s}^{t, \eta}\right)$, we choose $r>2 c_{1 / 2}$. Regarding the estimates (57) and (58), it is sufficient to choose $r>2 \max \left\{c_{1 / 2}, c_{3}\right\}$. Now, for the existence of the process $P$, it is sufficient to take $r>2 \max \left\{0, c_{5}, c_{3(2 m+1)-1}, c_{3 m-1}, c_{3 l-1}\right\}$ (for $p=\frac{3}{2}, q=3$ in (63)). Regarding Proposition 6, we have to add some restrictions originating from Lemma 1 throughout the proof. More precisely, it is sufficient to require $r>2 \max \left\{c_{7}, c_{2 m-1}, c_{3}\right\}$.

To conclude, the statement of the SMP holds true if the discount factor is chosen in a way such that all the previous results can be applied. Hence, it is sufficient to choose $k=1$ and $r$ such that $r>$ $64(2 m+1) \max \left\{0, c_{1 / 2}, c_{3}, c_{5}, c_{7}, c_{l-1}, c_{3 l-1}, c_{2 m-1}, c_{3 m-1}, c_{4 m-1}, c_{2(2 m+1)-1}, c_{3(2 m+1)-1}, c_{4(2 m+1)-1}\right\}$.

\section{REFERENCES}

[1] Seid Bahlali and Brahim Mezerdi. A general stochastic maximum principle for singular control problems. Electronic Journal of Probability, 10(30):988-1004, 2005.

[2] Ph. Briand, B. Delyon, Y. Hu, E. Pardoux, and L. Stoica. Lp solutions of backward stochastic differential equations. Stochastic Processes and their Applications, 108(1):109 - 129, 2003.

[3] Sandra Cerrai. Second order PDE's in finite and infinite dimension, volume 1762 of Lecture Notes in Mathematics. Springer-Verlag, Berlin, 2001. A probabilistic approach.

[4] Sonja Cox, Martin Hutzenthaler, and Arnulf Jentzen. Local lipschitz continuity in the initial value and strong completeness for nonlinear stochastic differential equations. preprint arXiv:1309.5595, 2013.

[5] Kai Du and Qingxin Meng. A maximum principle for optimal control of stochastic evolution equations. SIAM J. Control Optim., 51(6):4343-4362, 2013.

[6] Dufour F. and Miller B. Maximum principle for singular stochastic control problems. SIAM J. Control Optim., 45(2):668-698, 2006.

[7] Marco Fuhrman, Ying Hu, and Gianmario Tessitore. Stochastic maximum principle for optimal control of SPDEs. Appl. Math. Optim., 68(2):181-217, 2013. 
[8] Guangqiang Lan and Jiang-Lun Wu. New sufficient conditions of existence, moment estimations and non confluence for SDEs with non-Lipschitzian coefficients. Stochastic Process. Appl., 124(12):4030-4049, 2014.

[9] Qi Lü and Xu Zhang. General pontryagin-type stochastic maximum principle and backward stochastic evolution equations in infinite dimensions. preprint arXiv:1204.3275, 2012.

[10] Bohdan Maslowski and Petr Veverka. Sufficient stochastic maximum principle for discounted control problem. Appl. Math. Optim., 70(2):225-252, 2014.

[11] Bernt Øksendal and Agnès Sulem. Applied stochastic control of jump diffusions, volume 498. Springer, 2005.

[12] Bernt Øksendal and Agnès Sulem. Singular stochastic control and optimal stopping with partial information of Itô-Lévy processes. Research Report RR-7708, August 2011.

[13] Bernt Øksendal, Agnès Sulem, and Tusheng Zhang. Optimal control of stochastic delay equations and time-advanced backward stochastic differential equations. Adv. in Appl. Probab., 43(2):572-596, 032011.

[14] Martin Ondreját and Jan Seidler. On existence of progressively measurable modifications. Electron. Commun. Probab., 18:no. 20, 1-6, 2013.

[15] Carlo Orrieri. A stochastic maximum principle with dissipativity conditions. Disc. Cont. Dyn. Sist. A, to appear.

[16] Étienne Pardoux. BSDEs, weak convergence and homogenization of semilinear PDEs. In Nonlinear analysis, differential equations and control (Montreal, QC, 1998), volume 528 of NATO Sci. Ser. C Math. Phys. Sci., pages 503-549. Kluwer Acad. Publ., Dordrecht, 1999.

[17] Shige Peng. A general stochastic maximum principle for optimal control problems. SIAM J. Control Optim., 28(4):966-979, 1990.

[18] Shige Peng and Yufeng Shi. Infinite horizon forward-backward stochastic differential equations. Stochastic Process. Appl., 85(1):75-92, 2000.

[19] Claudia Prévôt and Michael Röckner. A concise course on stochastic partial differential equations, volume 1905 of Lecture Notes in Mathematics. Springer, Berlin, 2007.

[20] Shan Jian Tang and Xun Jing Li. Maximum principle for optimal control of distributed parameter stochastic systems with random jumps. In Differential equations, dynamical systems, and control science, volume 152 of Lecture Notes in Pure and Appl. Math., pages 867-890. Dekker, New York, 1994.

[21] Shan Jian Tang and Xun Jing Li. Necessary conditions for optimal control of stochastic systems with random jumps. SIAM Journal on Control and Optimization, 32(5):1447-1475, 1994.

[22] Gianmario Tessitore. Existence, uniqueness and space regularity of the adapted solutions of a backward SPDE. Stochastic Anal. Appl., 14(4):461-486, 1996.

[23] Zhen Wu and Feng Zhang. Maximum principle for stochastic recursive optimal control problems involving impulse controls. Abstr. Appl. Anal., 2012:1-16, 2012.

[24] Jiongmin Yong and Xun Yu Zhou. Stochastic Controls: Hamiltonian Systems and HJB Equations. New York: Springer-Verlag, 1999.

[25] Xun Yu Zhou. Stochastic near-optimal controls: Necessary and sufficient conditions for nearoptimality. SIAM J. Control Optim., 36(3):929-947, 1998.

(C. Orrieri) Dipartimento di Matematica, Universitì di Pavia. via Ferrata 1, 27100 Pavia, Italia

E-mail address: carlo.orrieri01@ateneopv.it

(P. Veverka) Dipartimento di Matematica, Politecnico di Milano. via Bonardi 9, 20133 Milano, Italia

E-mail address: petr.veverka@polimi.it 